\title{
EIGENVALUES AND LINEAR QUASIRANDOM HYPERGRAPHS
}

\author{
JOHN LENZ and DHRUV MUBAYI \\ Department of Mathematics, Statistics, and Computer Science, University of Illinois at Chicago, \\ 322 Science and Engineering Offices (M/C 249), 851 S. Morgan Street, Chicago, \\ IL 60607-7045, USA; \\ email: lenz@math.uic.edu,mubayi@math.uic.edu
}

Received 17 January 2013; accepted 28 July 2014

\begin{abstract}
Let $p(k)$ denote the partition function of $k$. For each $k \geqslant 2$, we describe a list of $p(k)-1$ quasirandom properties that a $k$-uniform hypergraph can have. Our work connects previous notions on linear hypergraph quasirandomness by Kohayakawa, Rödl, and Skokan, and by Conlon, Hàn, Person, and Schacht, and the spectral approach of Friedman and Wigderson. For each of the quasirandom properties that is described, we define the largest and the second largest eigenvalues. We show that a hypergraph satisfies these quasirandom properties if and only if it has a large spectral gap. This answers a question of Conlon, Hàn, Person, and Schacht. Our work can be viewed as a partial extension to hypergraphs of the seminal spectral results of Chung, Graham, and Wilson for graphs.
\end{abstract}

2010 Mathematics Subject Classification: 05C80 (primary); 05C50, 05 C65 (secondary)

\section{Introduction}

The study of quasirandom or pseudorandom graphs was initiated by Thomason $[43,44]$ and then refined by Chung et al. [15], resulting in a list of equivalent (deterministic) properties of graph sequences which are inspired by $G(n, p)$. Beginning with these foundational papers on the subject $[15,43,44]$, the last two decades have seen an explosive growth in the study of quasirandom structures in mathematics and computer science. For details on quasirandomness, we refer the reader to a survey of Krivelevich and Sudakov [30] for graphs, and recent papers of Gowers [23-25] for general quasirandom structures including hypergraphs.

(C) The Author(s) 2015. This is an Open Access article, distributed under the terms of the Creative Commons Attribution licence (http://creativecommons.org/licenses/by/3.0/), which permits unrestricted re-use, distribution, and reproduction in any medium, provided the original work is properly cited. 
1.1. Previous results. The core of what Chung et al. [15] proved is that several properties of graph sequences are equivalent. Two of them are Disc and Count $[A \perp l]$. The first states that all sufficiently large vertex sets have the same edge density as the original graph, and the second states that for all fixed graphs $F$ the number of copies of $F$ is what one would expect in a random graph with the same density.

A $k$-uniform hypergraph is a pair of finite sets $(V(H), E(H))$, where $E(H) \subseteq$ $\left(\begin{array}{c}V(H) \\ k\end{array}\right)$ is a collection of $k$-subsets of $V(H)$. For $U \subseteq V(H)$, the induced subgraph on $U$, denoted $H[U]$, is the hypergraph with vertex set $U$ and edge set $\{e \in$ $E(H): e \subseteq U\}$. If $F$ and $G$ are hypergraphs, a labeled copy of $F$ in $H$ is an edgepreserving injection $V(F) \rightarrow V(H)$; that is, an injection $\alpha: V(F) \rightarrow V(H)$ such that, if $E$ is an edge of $F$, then $\{\alpha(x): x \in E\}$ is an edge of $H$. A graph is a 2-uniform hypergraph.

Almost immediately after proving their theorem, Chung and Graham $[8,9,12-$ 14] investigated generalizing the theorem to $k$-uniform hypergraphs. One initial difficulty in generalizing quasirandomness to $k>2$ is an observation by Rödl that a construction of Erdôs and Hajnal [18] shows that the hypergraph generalizations of $\mathrm{Disc}$ and Count $[\mathrm{All}]$ are not equivalent. Motivated by this, Chung and Graham [9, 12-14] investigated how to strengthen the property $\mathrm{Disc}$ to make it equivalent to Count $[\mathrm{Al} 1]$. They found several properties equivalent to Count $[\mathrm{A} I 1]$; the main property they use is related to the number of even/odd subgraphs of a given hypergraph, which they called Deviation. Simultaneously, Frankl and Rödl [20] also obtained a property stronger than $\mathrm{Disc}$ which is equivalent to Count $[\mathrm{All}]$. Subsequently, other properties equivalent to Count $[A I l]$ have been studied by several researchers $[6,23,27$, 29].

It remained open whether the simpler property Disc for $k$-uniform hypergraphs is equivalent to counting some class of hypergraphs or counting a single substructure. This is related to the weak hypergraph regularity lemma [10, 20, 39]. Recently, Kohayakawa et al. [28] answered this question by showing that $\mathrm{Disc}$ is equivalent to counting the family of linear hypergraphs, where a hypergraph $H$ is linear if every pair of distinct edges share at most one vertex. Building on this, Conlon et al. [16] showed that Disc is equivalent to counting a type of linear four cycle. These two results can be combined into the following theorem.

THEOREM 1 (Kohayakawa-Nagle-Rödl-Schacht [28] and Conlon-Hàn-PersonSchacht [16]). Let $0<p<1$ be a fixed constant, and let $\mathcal{H}=\left\{H_{n}\right\}_{n \rightarrow \infty}$ be a sequence of $k$-uniform hypergraphs such that $\left|V\left(H_{n}\right)\right|=n$ and $\left|E\left(H_{n}\right)\right| \geqslant$ $p\left(\begin{array}{l}n \\ k\end{array}\right)+o\left(n^{k}\right)$. The following properties are equivalent. 
- Disc: For every $U \subseteq V\left(H_{n}\right),\left|E\left(H_{n}[U]\right)\right|=p\left(\begin{array}{c}|U| \\ k\end{array}\right)+o\left(n^{k}\right)$.

- Count [Iinear]: For every fixed linear k-uniform hypergraph $F$ with $e$ edges and $f$ vertices, the number of labeled copies of $F$ in $H_{n}$ is $p^{e} n^{f}+o\left(n^{f}\right)$.

- Cycle $e_{4}$ : The number of labeled copies of $C_{4}$ in $H_{n}$ is at most $p^{\left|E\left(C_{4}\right)\right|} n^{\left|V\left(C_{4}\right)\right|}+$ $o\left(n^{\left|V\left(C_{4}\right)\right|}\right)$, where $C_{4}$ is a linear hypergraph defined precisely in Section 2.

Note that Conlon et al. [16] put the condition that $\left|E\left(H_{n}\right)\right| \geqslant p\left(\begin{array}{l}n \\ k\end{array}\right)+o\left(n^{k}\right)$ into the statement of the properties that do not trivially imply it like Disc, and this is equivalent to the way we have stated Theorem 1. Conlon et al. [16] have several more properties including, induced subgraph counts and common neighborhood sizes, but we consider the properties stated in Theorem 1 as the core properties.

1.2. Our results. Another graph property equivalent to Disc is Eig, which states that, if $\mu_{1}$ and $\mu_{2}$ are the first and second largest (in absolute value) eigenvalues of the adjacency matrix of the graph, respectively, then $\mu_{2}=o\left(\mu_{1}\right)$. Neither Chung and Graham [9, 12-14] nor Kohayakawa et al. [29] provided a generalization of Eig to hypergraphs. Later, Conlon et al. [16] asked whether there exists a generalization of Eig to $k$-uniform hypergraphs which is equivalent to Disc. The eigenvalue description of graph quasirandomness has proved to be a very useful result to show that certain explicitly constructed graphs are quasirandom (see $[3,4,33,41]$ ).

This leads to our first main contribution. We define a generalization of $E$ ig to $k$-uniform hypergraphs and add it into the equivalences stated in Theorem 1. This answers the aforementioned question of Conlon et al. [16].

Our second contribution is to generalize Theorem 1 to a slightly larger class of hypergraphs. Let $k \geqslant 2$ be an integer, and let $\pi$ be a proper partition of $k$, by which we mean that $\pi$ is an unordered list of at least two positive integers whose sum is $k$. For the partition $\pi$ of $k$ given by $k=k_{1}+\cdots+k_{t}$, we will abuse notation by saying that $\pi=k_{1}+\cdots+k_{t}$. For every proper partition $\pi$, we define properties $\operatorname{Expand}[\pi], \operatorname{Eig}[\pi]$, and $\mathrm{CyCle}_{4}[\pi]$, and show that they are equivalent.

DEFINITION. Let $k \geqslant 2$, and let $\pi=k_{1}+\cdots+k_{t}$ be a proper partition of $k$. A $k$-uniform hypergraph $F$ is $\pi$-linear if there exists an ordering $E_{1}, \ldots, E_{m}$ of the edges of $F$ such that, for every $i$, there exists a partition of the vertices of $E_{i}$ into $A_{i, 1}, \ldots, A_{i, t}$ such that, for $1 \leqslant s \leqslant t,\left|A_{i, s}\right|=k_{s}$, and for every $j<i$, there exists an $s$ such that $E_{j} \cap E_{i} \subseteq A_{i, s}$.

Our hypergraph eigenvalues are based on definitions of Friedman and Wigderson [21, 22] (see Section 3). In graphs, it is easier to study the 
eigenvalues of regular graphs (possibly with loops). A similar situation occurs for hypergraphs, so Friedman and Wigderson [21, 22] focused almost exclusively on the following notion of regular for hypergraphs.

DEFINITION. A $k$-uniform hypergraph with loops $H$ consists of a finite set $V(H)$ and a collection $E(H)$ of $k$-element multisets of elements from $V(H)$. Informally, every edge has size exactly $k$, but a vertex is allowed to be repeated inside of an edge. A $k$-uniform hypergraph with loops $H$ is $d$-coregular if, for every $(k-1)$ multiset $S$, there are exactly $d$ edges which contain $S$.

The following is our main theorem.

THEOREM 2 (Main result). Let $0<p<1$ be a fixed constant, and let $\mathcal{H}=$ $\left\{H_{n}\right\}_{n \rightarrow \infty}$ be a sequence of $k$-uniform hypergraphs with loops such that $\left|V\left(H_{n}\right)\right|=$ $n$ and $H_{n}$ is $\lfloor p n\rfloor$-coregular. Let $\pi=k_{1}+\cdots+k_{t}$ be a proper partition of $k$. The following properties are equivalent.

- $\operatorname{Eig}[\pi]: \lambda_{1, \pi}\left(H_{n}\right)=p n^{k / 2}+o\left(n^{k / 2}\right)$ and $\lambda_{2, \pi}\left(H_{n}\right)=o\left(n^{k / 2}\right)$, where $\lambda_{1, \pi}\left(H_{n}\right)$ and $\lambda_{2, \pi}\left(H_{n}\right)$ are the first and second largest eigenvalues of $H_{n}$ with respect to $\pi$, which are defined in Section 3.

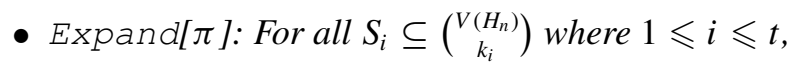

$$
e\left(S_{1}, \ldots, S_{t}\right)=p \prod_{i=1}^{t}\left|S_{i}\right|+o\left(n^{k}\right),
$$

where $e\left(S_{1}, \ldots, S_{t}\right)$ is the number of tuples $\left(s_{1}, \ldots, s_{t}\right)$ such that $s_{1} \cup \cdots \cup s_{t}$ is a hyperedge and $s_{i} \in S_{i}$.

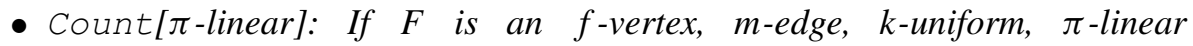
hypergraph, then the number of labeled copies of $F$ in $H_{n}$ is $p^{m} n^{f}+o\left(n^{f}\right)$.

- $\mathrm{CyCle}_{4}[\pi]$ : The number of labeled copies of $C_{\pi, 4}$ in $H_{n}$ is at most $p^{\left|E\left(C_{\pi, 4}\right)\right|} n^{\left|V\left(C_{\pi, 4}\right)\right|}+o\left(n^{\left|V\left(C_{\pi, 4}\right)\right|}\right)$, where $C_{\pi, 4}$ is the hypergraph four cycle of type $\pi$ defined in Section 2.

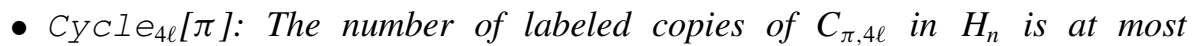

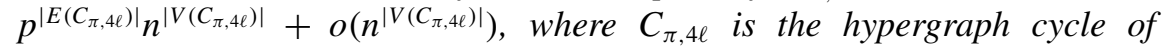
type $\pi$ and length $4 \ell$ defined in Section 2.

In fact, all implications above except $C y C l e_{4 \ell}[\pi] \Rightarrow E i g[\pi]$ are true with the coregular condition replaced by the weaker condition that $\left|E\left(H_{n}\right)\right| \geqslant p\left(\begin{array}{l}n \\ k\end{array}\right)+$ $o\left(n^{k}\right)$. 


\section{REMARKS.}

- In a companion paper [32], we prove that $\mathrm{CyCle}_{4 \ell}[\pi] \Rightarrow \mathrm{Eig}[\pi]$ for all sequences $\mathcal{H}=\left\{H_{n}\right\}_{n \rightarrow \infty}$, where $H_{n}$ is a $k$-uniform hypergraph with loops, $\left|V\left(H_{n}\right)\right|=n$, and $\left|E\left(H_{n}\right)\right| \geqslant p\left(\begin{array}{l}n \\ k\end{array}\right)+o\left(n^{k}\right)$.

- Following Chung et al. [15], our results extend to sequences which are not defined for every $n$ as follows. Let $\mathcal{H}=\left\{H_{n_{q}}\right\}_{q \rightarrow \infty}$ be a sequence of hypergraphs such that $\left|V\left(H_{n_{q}}\right)\right|=n_{q}, n_{q}<n_{q+1}$, and $\left|E\left(H_{n_{q}}\right)\right| \geqslant p\left(\begin{array}{c}n_{q} \\ k\end{array}\right)+o\left(n_{q}^{k}\right)$, where now the little- $o$ expression means there exists a function $f(q)$ such that $\left|E\left(H_{n_{q}}\right)\right| \geqslant p\left(\begin{array}{c}n_{q} \\ k\end{array}\right)+f(q)$ with $\lim _{q \rightarrow \infty} f(q) n_{q}^{-k}=0$. Similarly, when we say that property $P$ (which might include a little- $o$ expression) implies a property $P^{\prime}$, what we mean is that there exist functions $f(q)$ and $f^{\prime}(q)$ such that $P(f(q))$ implies $P^{\prime}\left(f^{\prime}(q)\right)$, where the notation $P(f(q))$ stands for the property $P$ with the little- $o$ replaced by the function $f(q)$.

- If $\pi=1+\cdots+1$, the partition of $k$ into $k$ ones, then the equivalences Expand $[\pi] \Leftrightarrow \operatorname{Count}[\pi$-linear $] \Leftrightarrow \mathrm{CyCl}_{4}[\pi]$ of Theorem 2 constitute Theorem 1 . Therefore, the property $\operatorname{Eig}[1+\cdots+1]$ is the spectral property that is equivalent to the weak quasirandom properties studied by Kohayakawa et al. [29] and Conlon et al. [16].

- If $\pi^{\prime}$ is a refinement of $\pi$, then clearly Count [ $\pi$-linear] $\Rightarrow$ Count [ $\pi^{\prime}$-linear], and so, if $\left\{H_{n}\right\}_{n \rightarrow \infty}$ is a sequence satisfying the properties in Theorem 2 for $\pi$, it satisfies the properties for $\pi^{\prime}$. In a companion paper [34], we show the converse: if $\pi^{\prime}$ is not a refinement of $\pi$, then $\operatorname{Expand}[\pi] \not \Rightarrow \operatorname{Expand}\left[\pi^{\prime}\right]$, so the property Expand $[\pi]$ is distinct for distinct $\pi$ and arranged in a poset via partition refinement.

The remainder of this paper is organized as follows. In Section 2, we define the hypergraph cycles $C_{\pi, 4}$. Section 3 gives the formal definition of eigenvalues with respect to $\pi$. Theorem 2 is proved by showing a chain of implications in the order stated in the theorem; Section 4 proves $\operatorname{Eig}[\pi] \Rightarrow \operatorname{Expand}[\pi]$, Section 5 proves Expand $[\pi] \Rightarrow$ Count $\left[\pi\right.$-linear], and Section 6 shows that $\mathrm{Cycle}_{4 \ell}[\pi]$ $\Rightarrow \operatorname{Eig}[\pi]$ for $d$-coregular hypergraphs with loops. Throughout this paper, we use the notation $[n]=\{1, \ldots, n\}$.

\section{Hypergraph cycles}

In this section, we define the hypergraph cycles $C_{\pi, 2 \ell}$. The hypergraph cycles $C_{\pi, 2 \ell}$ are defined by first defining steps, then defining a path as a combination of steps, and finally defining the cycle as a path with its endpoints identified. 


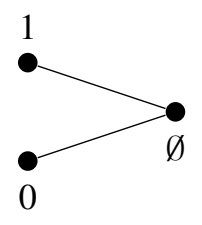

$A$

(a) $S_{(1,1)}$

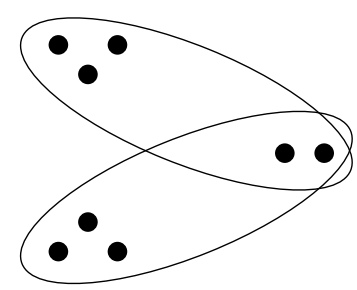

$A$

(b) $S_{(3,2)}$

Figure 1: Steps with $t=2$.

Definition. Let $\vec{\pi}=(1, \ldots, 1)$ be the ordered partition of $t$ into $t$ parts. Define the step of type $\vec{\pi}$, denoted $S_{\vec{\pi}}$, as follows. Let $A$ be a vertex set of size $2^{t-1}$ where elements are labeled by binary strings of length $t-1$, and let $B_{2}, \ldots, B_{t}$ be disjoint sets of size $2^{t-2}$ where elements are labeled by binary strings of length $t-2$. The vertex set of $S_{\vec{\pi}}$ is the disjoint union $A \dot{\cup} B_{2} \dot{\cup} \ldots \dot{\cup} B_{t}$. Make $\left\{a, b_{2}, \ldots\right.$, $\left.b_{t}\right\}$ a hyperedge of $S_{\vec{\pi}}$ if $a \in A, b_{j} \in B_{j}$, and the code for $b_{j+1}$ is equal to the code formed by removing the $j$ th bit of the code for $a$.

For a general $\vec{\pi}=\left(k_{1}, \ldots, k_{t}\right)$, start with $S_{(1, \ldots, 1)}$ and enlarge each vertex into the appropriate size; that is, a vertex in $A$ is expanded into $k_{1}$ vertices, and each vertex in $B_{j}$ is expanded into $k_{j}$ vertices. More precisely, the vertex set of $S_{\vec{\pi}}$ is $\left(A \times\left[k_{1}\right]\right) \dot{\cup}\left(B_{2} \times\left[k_{2}\right]\right) \dot{\cup} \cdots \dot{\cup}\left(B_{t} \times\left[k_{t}\right]\right)$, and, if $\left\{a, b_{2}, \ldots, b_{t}\right\}$ is an edge of $S_{(1, \ldots, 1)}$, then $\left\{(a, 1), \ldots,\left(a, k_{1}\right),\left(b_{2}, 1\right), \ldots,\left(b_{2}, k_{2}\right), \ldots,\left(b_{t}, 1\right), \ldots,\left(b_{t}, k_{t}\right)\right\}$ is a hyperedge of $S_{\vec{\pi}}$.

This defines the step of type $\vec{\pi}$, denoted $S_{\vec{\pi}}$. Let $A^{(0)}$ be the ordered tuple of vertices of $A$ in $S_{\vec{\pi}}$ whose binary code ends with zero, and let $A^{(1)}$ be the ordered tuple of vertices of $A$ whose binary code ends with one, where vertices are listed in lexicographic order within each $A^{(i)}$. These tuples $A^{(0)}$ and $A^{(1)}$ are the two attach tuples of $S_{\vec{\pi}}$

Figure 1 shows the steps of type $(1,1)$ and type $(3,2)$. Notice that each step has 'length' two if we consider the attach tuples as the 'ends' of a path.

Figure 2 shows two different drawings of the step of type $\vec{\pi}=(1,1,1)$. Notice that the attach tuples are easily visible in Figure 2(b), since the two attach tuples are the codes in $A$ ending with a zero and a one. The step of type $\vec{\pi}=\left(k_{1}, k_{2}, k_{3}\right)$ is an enlarged version of Figure 2 similar to Figure 1(b).

In general, for arbitrary $\vec{\pi}$, the step $S_{\vec{\pi}}$ can be drawn in two ways similar to Figure 2. First, from the definition, a step is a $k$-partite hypergraph with parts $A$, 


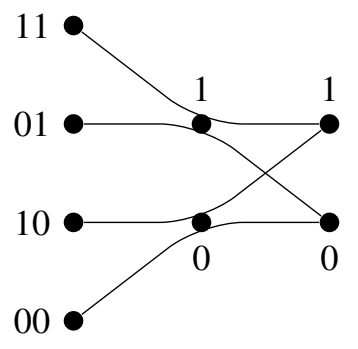

$A \quad B_{2} \quad B_{3}$

(a) $S_{(1,1,1)}$

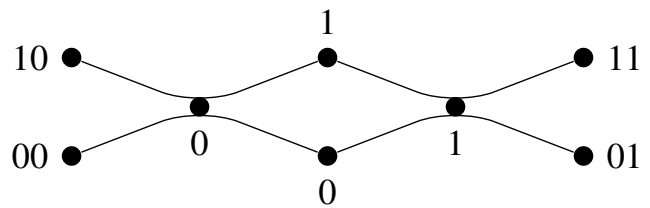

$A^{(0)}$

$B_{2}$

$B_{3}$

$B_{2}$

$A^{(1)}$

Figure 2: Steps of type $\pi=(1,1,1)$.

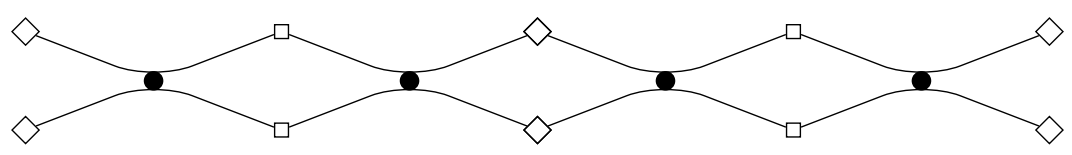

Figure 3: $P_{(1,1,1), 4}$.

$B_{2}, \ldots, B_{t}$, so it can be drawn similar to Figure 2(a). But the step can also be drawn with the two attach tuples on separate ends of the picture like Figure 2(b). Let $M_{0}$ be the set of edges incident to vertices in the attach tuple $A^{(0)}$, and let $M_{1}$ be the set of edges incident to vertices in $A^{(1)}$. Edges from $M_{0}$ and $M_{1}$ intersect only in vertices in $B_{t}$ because, if $a_{0} \in A^{(0)}$ and $a_{1} \in A^{(1)}$, then the code for $a_{0}$ ends in a zero and the code for $a_{1}$ ends in a one, so only when deleting the last bit will the codes possibly be the same. Therefore, the step $S_{\vec{\pi}}$ can be viewed as a type of length-two path in a hypergraph formed from a collection of $k$-partite edges $M_{0}$ between $A^{(0)}$ and $B_{t}$ and another collection of $k$-partite edges $M_{1}$ between $B_{t}$ and $A^{(1)}$.

Definition. Let $\ell \geqslant 1$. The path of type $\vec{\pi}$ of length $2 \ell$, denoted $P_{\vec{\pi}, 2 \ell}$, is the hypergraph formed from $\ell$ copies of $S_{\vec{\pi}}$ with successive attach tuples identified. That is, let $T_{1}, \ldots, T_{\ell}$ be copies of $S_{\vec{\pi}}$, and let $A_{i}^{(0)}$ and $A_{i}^{(1)}$ be the attach tuples of $T_{i}$. The hypergraph $P_{\vec{\pi}, 2 \ell}$ is the hypergraph consisting of $T_{1}, \ldots, T_{\ell}$, where the vertices of $A_{i}^{(1)}$ are identified with $A_{i+1}^{(0)}$ for every $1 \leqslant i \leqslant \ell-1$. (Recall that, by definition, $A_{i}^{(1)}$ and $A_{i+1}^{(0)}$ are tuples (that is ordered lists) of vertices, so the identification of $A_{i}^{(1)}$ and $A_{i+1}^{(0)}$ identifies the corresponding vertices in these tuples.) The attach tuples of $P_{\vec{\pi}, 2 \ell}$ are the tuples $A_{1}^{(0)}$ and $A_{\ell}^{(1)}$. 
In Figure 3, the path $P_{(1,1,1), 4}$ is drawn as two copies of $S_{(1,1,1)}$ with attach tuples identified. The diamond, circle, and square vertices keep track of the parts $A, B_{2}$, $B_{3}$. For a general $P_{\left(k_{1}, k_{2}, k_{3}\right), 4}$, each diamond vertex is enlarged into $k_{1}$ vertices, each circle vertex is enlarged into $k_{2}$ vertices, and each square vertex is enlarged into $k_{3}$ vertices. For a general $\vec{\pi}$, every step can be visualized as in Figure 2(b) as two collections of $k$-partite edges $M_{0}$ and $M_{1}$ between $A$ and $B_{t}$, so all paths $P_{\vec{\pi}, 2 \ell}$ can be visualized as in Figure 3 as a concatenation of steps.

Definition. Let $\ell \geqslant 2$. The cycle of type $\pi$ and length $2 \ell$, denoted $C_{\pi, 2 \ell}$, is the hypergraph formed by picking any ordering $\vec{\pi}$ of $\pi$ and identifying the attach tuples of $P_{\vec{\pi}, 2 \ell}$.

The definition of $C_{\pi, 2 \ell}$ is independent of the ordering $\vec{\pi}$; a proof appears in [31].

Definition. Let $\ell \geqslant 2$. A walk of type $\vec{\pi}$ and length $2 \ell$ in a hypergraph $H$ is a function $f: V\left(P_{\vec{\pi}, 2 \ell}\right) \rightarrow V(H)$ that preserves edges. Informally, a walk is a path where the vertices are not necessarily distinct. A circuit of type $\pi$ of length $2 \ell$ in a hypergraph $H$ is a function $f: V\left(C_{\pi, 2 \ell}\right) \rightarrow V(H)$ that preserves edges. Informally, a circuit is a cycle where the vertices are not necessarily distinct.

There are two alternative definitions of the cycle of length four. First, Conlon et al. [16] defined a cycle of length four for $\pi=1+\cdots+1$ by an operation called reflection. Our definition of $C_{1+\cdots+1,4}$ is equivalent to the definition in [16]; this can be seen by noticing that the bit strings in our definition keep track of the vertex duplications which occur during reflection.

Finally, there is a concise direct definition of the cycle of type $\pi$ and length four which avoids the complexity of defining steps and paths. We will not use this shorter definition in this paper, instead working with steps, paths, and walks, but we include this short definition for completeness. Let $D_{1}, \ldots, D_{t}$ be disjoint sets of size $2^{t-1}$ whose elements are labeled by $(t-1)$-length binary strings. The vertex set is $D_{1} \dot{\cup} \ldots \dot{\cup} D_{t}$. For $d_{1} \in D_{1}, \ldots, d_{t} \in D_{t}$, make $\left\{d_{1}, \ldots, d_{t}\right\}$ a hyperedge if there exists a binary string $s$ of length $t$ such that the code for $d_{i}$ equals the code formed by deleting the $i$ th bit of $s$. The cycle for general $\pi$ is formed by enlarging this cycle appropriately. Figure 4 shows cycles drawn using this definition.

\section{Hypergraph eigenvalues}

This section contains the definition of the largest and second largest eigenvalues of a hypergraph with respect to $\pi$, and it also contains some discussion and basic facts about them. 


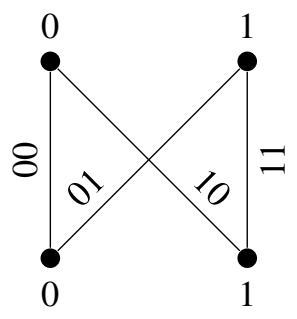

(a) $C_{(1,1), 4}$

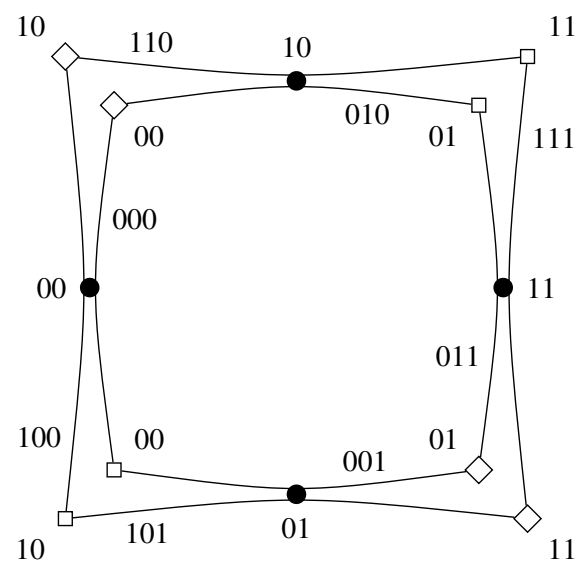

(b) $C_{(1,1,1), 4}$

Figure 4: Alternate definition of the cycle of length four.

There have been three independently developed approaches to hypergraph eigenvalues: a definition by Chung [11] and Lu and Peng [35, 36] using matrices, an approach of Friedman and Wigderson [21, 22] and Cooper and Dutle [17], and lastly the eigenvalues of the shadow graph $[7,19,37,38,40]$. The definitions of Friedman and Wigderson [21, 22] are most suitable for our purposes, and we will use their definitions as our starting point.

Definition. Let $V_{1}, \ldots, V_{k}$ be finite-dimensional vector spaces over $\mathbb{R}$. A $k$ linear map is a function $\phi: V_{1} \times \cdots \times V_{k} \rightarrow \mathbb{R}$ such that, for each $1 \leqslant i \leqslant k$, $\phi$ is linear in the $i$ th coordinate. That is, for every fixed $x_{i} \in V_{i}, \phi\left(x_{1}, \ldots, x_{i-1}, \cdot, x_{i+1}\right.$, $\left.\ldots, x_{n}\right)$ is a linear map from $V_{i}$ to $\mathbb{R}$. A $k$-linear map $\phi: V^{k} \rightarrow \mathbb{R}$ is symmetric if for all permutations $\eta$ of $[k]$ and all $x_{1}, \ldots, x_{k} \in V, \phi\left(x_{1}, \ldots, x_{k}\right)=\phi\left(x_{\eta(1)}, \ldots\right.$, $\left.x_{\eta(k)}\right)$.

Definition. Let $V_{1}, \ldots, V_{k}$ be finite-dimensional vector spaces over $\mathbb{R}$, let $B_{i}=$ $\left\{b_{i, 1}, \ldots, b_{i, \mathrm{dim}\left(V_{i}\right)}\right\}$ be an orthonormal basis of $V_{i}$, and let $\phi: B_{1} \times \cdots \times B_{k} \rightarrow \mathbb{R}$ be any map. Extending $\phi$ linearly to $V_{1} \times \cdots \times V_{k}$ means that $\phi$ is extended to a map $V_{1} \times \cdots \times V_{k} \rightarrow \mathbb{R}$, where, for $x_{1} \in V_{1}, \ldots, x_{k} \in V_{k}$,

$$
\phi\left(x_{1}, \ldots, x_{k}\right)=\sum_{j_{1}=1}^{\operatorname{dim}\left(V_{1}\right)} \cdots \sum_{j_{k}=1}^{\operatorname{dim}\left(V_{k}\right)}\left\langle x_{1}, b_{1, j_{1}}\right\rangle \cdots\left\langle x_{k}, b_{k, j_{k}}\right\rangle \phi\left(b_{1, j_{1}}, \ldots, b_{k, j_{k}}\right) .
$$

Note that extending $\phi$ in this way produces a $k$-linear map. 
DEFINITION (Friedman and Wigderson [21, 22]). Let $H$ be a $k$-uniform hypergraph with loops. The adjacency map of $H$ is the symmetric $k$-linear map $\tau_{H}: W^{k} \rightarrow \mathbb{R}$ defined as follows, where $W$ is the vector space over $\mathbb{R}$ of dimension $|V(H)|$. First, for all $v_{1}, \ldots, v_{k} \in V(H)$, let

$$
\tau_{H}\left(e_{v_{1}}, \ldots, e_{v_{k}}\right)= \begin{cases}1 & \left\{v_{1}, \ldots, v_{k}\right\} \in E(H), \\ 0 & \text { otherwise },\end{cases}
$$

where $e_{v}$ denotes the indicator vector of the vertex $v$, that is the vector which has a one in coordinate $v$ and zero in all other coordinates. We have defined the value of $\tau_{H}$ when the inputs are standard basis vectors of $W$. Extend $\tau_{H}$ to all the domain linearly.

Definition. Let $W_{1}, \ldots, W_{k}$ be finite-dimensional vector spaces over $\mathbb{R}$, let $\|\cdot\|$ denote the Euclidean 2-norm on $W_{i}$, and let $\phi: W_{1} \times \cdots \times W_{k} \rightarrow \mathbb{R}$ be a $k$-linear map. The spectral norm of $\phi$ is

$$
\|\phi\|=\sup _{\substack{x_{i} \in W_{i} \\\left\|x_{i}\right\|=1}}\left|\phi\left(x_{1}, \ldots, x_{k}\right)\right| .
$$

Before defining the first and second largest eigenvalues of $H$ with respect to a general partition $\pi$, we give the definitions when $\pi=1+\cdots+1$, that is $\pi$ is the partition into $k$ ones.

DEFINITION. Let $H$ be an $n$-vertex, $k$-uniform hypergraph, let $W$ be the vector space over $\mathbb{R}$ of dimension $n$, and let $J: W^{k} \rightarrow \mathbb{R}$ be the all-ones map. That is, if $e_{i_{1}}, \ldots, e_{i_{k}}$ are any standard basis vectors of $W$, then $J\left(e_{i_{1}}, \ldots, e_{i_{k}}\right)=1$, and $J$ is extended linearly to all of the domain as in (1).

The largest eigenvalue of $H$ with respect to $\pi=1+\cdots+1$ is $\left\|\tau_{H}\right\|$, and the second largest eigenvalue of $H$ with respect to $\pi=1+\cdots+1$ is $\left\|\tau_{H}-\frac{k !|E(H)|}{n^{k}} J\right\|$.

In order to extend this definition to general $\vec{\pi}=\left(k_{1}, \ldots, k_{t}\right)$, it is convenient to use the language of tensor products.

DEFINITION. Let $V$ and $W$ be finite-dimensional vector spaces over $\mathbb{R}$ of dimension $n$ and $m$, respectively. The tensor product of $V$ and $W$, written $V \otimes W$, is the vector space over $\mathbb{R}$ of dimension $\mathrm{nm}$. A typical tensor $a$ in $V \otimes W$ has the form $a=\sum_{i=1}^{\operatorname{dim}(V)} \sum_{j=1}^{\operatorname{dim}(W)} \alpha_{i, j}\left(e_{i} \otimes e_{j}^{\prime}\right)$, where $\alpha_{i, j} \in \mathbb{R}, e_{1}, \ldots, e_{\operatorname{dim}(V)}$ is the standard basis of $V$, and $e_{1}^{\prime}, \ldots, e_{\operatorname{dim}(W)}^{\prime}$ is the standard basis of $W$. The length of a tensor is the length of the vector in the vector space $V \otimes W$. Thus the length of $a$ is $\left(\sum_{i=1}^{\operatorname{dim}(V)} \sum_{j=1}^{\operatorname{dim}(W)} \alpha_{i, j}^{2}\right)^{1 / 2}$. 
We are now ready to define the map $\tau_{\vec{\pi}}$ and then the first and second largest eigenvalues of $H$ with respect to $\pi$ for a general $\pi$. In the definition, think of the tensor product $W^{\otimes k_{i}}$ as a vector space of dimension $|V(H)|^{k_{i}}$ indexed by ordered $k_{i}$-sets of vertices.

DEFINITION. Let $W$ be a finite-dimensional vector space over $\mathbb{R}$, let $\sigma: W^{k} \rightarrow \mathbb{R}$ be any $k$-linear function, and let $\vec{\pi}$ be a proper ordered partition of $k$, so $\vec{\pi}=\left(k_{1}\right.$, $\ldots, k_{t}$ ) for some integers $k_{1}, \ldots, k_{t}$ with $t \geqslant 2$. Now define a $t$-linear function $\sigma_{\vec{\pi}}: W^{\otimes k_{1}} \times \cdots \times W^{\otimes k_{t}} \rightarrow \mathbb{R}$ by first defining $\sigma_{\vec{\pi}}$ when the inputs are basis vectors of $W^{\otimes k_{i}}$ and then extending linearly. For each $i, B_{i}=\left\{b_{i, 1} \otimes \cdots \otimes b_{i, k_{i}}\right.$ : $b_{i, j}$ is a standard basis vector of $\left.W\right\}$ is a basis of $W^{\otimes k_{i}}$, so, for each $i$, pick $b_{i, 1} \otimes$ $\cdots \otimes b_{i, k_{i}} \in B_{i}$ and define

$$
\sigma_{\vec{\pi}}\left(b_{1,1} \otimes \cdots \otimes b_{1, k_{1}}, \ldots, b_{t, 1} \otimes \cdots \otimes b_{t, k_{t}}\right)=\sigma\left(b_{1,1}, \ldots, b_{1, k_{1}}, \ldots, b_{t, 1}, \ldots, b_{t, k_{t}}\right) .
$$

Now extend $\sigma_{\vec{\pi}}$ linearly to all of the domain. $\sigma_{\vec{\pi}}$ will be $t$-linear since $\sigma$ is $k$-linear.

DEFINITION. Let $H$ be a $k$-uniform hypergraph with loops, and let $\tau=\tau_{H}$ be the ( $k$-linear) adjacency map of $H$. Let $\pi$ be any (unordered) partition of $k$ and let $\vec{\pi}$ be any ordering of $\pi$. The largest and second largest eigenvalues of $H$ with respect to $\pi$, denoted $\lambda_{1, \pi}(H)$ and $\lambda_{2, \pi}(H)$, are defined as

$$
\lambda_{1, \pi}(H):=\left\|\tau_{\vec{\pi}}\right\| \quad \text { and } \quad \lambda_{2, \pi}(H):=\left\|\tau_{\vec{\pi}}-\frac{k !|E(H)|}{n^{k}} J_{\vec{\pi}}\right\| .
$$

Both $\lambda_{1, \pi}(H)$ and $\lambda_{2, \pi}(H)$ are well defined since, for any two orderings $\vec{\pi}$ and $\vec{\pi}^{\prime}$ of $\pi, \tau_{\vec{\pi}}=\tau_{\vec{\pi}^{\prime}}$ and $J_{\vec{\pi}}=J_{\vec{\pi}^{\prime}}$ since both $\tau$ and $J$ are symmetric maps.

\section{REMARKS.}

- For a graph $G(k=2$ and $\pi=1+1), \lambda_{1,1+1}(G)$ equals the largest eigenvalue in absolute value of the adjacency matrix $A$ of $G$ since both are equal to $\sup \left\{\left|x^{T} A x\right|:\|x\|=1\right\}$. Additionally, if $G$ is $d$-regular, then $\lambda_{2,1+1}(G)$ equals the second largest eigenvalue of $A$ in absolute value. Indeed, if $G$ is a $d$-regular graph, then $2|E(H)| / n^{2}=d / n$, so $\lambda_{2,1+1}(G)=\left\|\tau_{G}-(d / n) J\right\|$. The bilinear map $\tau_{G}-(d / n) J$ corresponds to the matrix $A-(d / n) J$, where $J$ is now the all-ones matrix. The largest eigenvalue of $A-(d / n) J$ in absolute value is the second largest eigenvalue of $A$ in absolute value, and this equals the spectral norm of the respective map.

- For any $k$-uniform hypergraph $H, \lambda_{1,1+\cdots+1}(H)$ exactly matches the definition of Friedman and Wigderson [21, 22]. [21, 22] did not define the second 
largest eigenvalue for all hypergraphs. For $d$-coregular hypergraphs with loops, $[21,22]$ defined the second largest eigenvalue, and it exactly corresponds to our definition of $\lambda_{2,1+\cdots+1}(H)$, where $k !|E(H)| / n^{k}=d / n$ (recall that $H$ has loops, which is why $n^{k}$ appears in the denominator instead of the falling factorial). For the random hypergraph $G^{(k)}(n, p),[21,22]$ also defined a second largest eigenvalue with respect to density $p$ as the spectral norm of $\tau_{G(n, p)}-p J$. While different from our definition, $p=(1+o(1)) k !|E(G(n, p))| / n^{k}$, so the definitions are similar.

- If $H$ is a $k$-uniform, $d$-coregular hypergraph with loops, Friedman and Wigderson [21, 22] proved several facts about $\lambda_{1,1+\cdots+1}(H)$ and $\lambda_{2,1+\cdots+1}(H)$. First, $\lambda_{1,1+\cdots+1}(H)=d n^{(k-2) / 2}$, and the supremum is achieved by the allones vectors scaled to unit length. They also proved several facts about $\lambda_{2,1+\cdots+1}(H)$, including upper and lower bounds, an expander mixing lemma which we generalize to all $\pi$ in Theorem 3 , and the asymptotic value of $\lambda_{2,1+\cdots+1}(G(n, p))$.

\section{4. $\operatorname{Eig}[\pi] \Rightarrow \operatorname{Expand}[\pi]$}

In this section, we prove a generalization of the graph expander mixing lemma which relates spectral and expansion properties of graphs. The graph version was first discovered independently by Alon and Milman [2] and Tanner [42]. For background on graph expansion and eigenvalues, see $[1,5,26]$. The following theorem extends the hypergraph expander mixing lemma of Friedman and Wigderson [21, 22], which applied for $\pi=1+\cdots+1$. The theorem is stated for ordered partitions $\vec{\pi}$, but trivially gives the same result for any ordering $\vec{\pi}$ of a partition $\pi$.

THEOREM 3 (Hypergraph expander mixing lemma). Let $H$ be an n-vertex, $k$-uniform hypergraph with loops. Let $\vec{\pi}=\left(k_{1}, \ldots, k_{t}\right)$ be a proper ordered partition of $k$, and let $S_{i} \subseteq\left(\begin{array}{c}V(H) \\ k_{i}\end{array}\right)$ for $1 \leqslant i \leqslant t$ (where the elements of $S_{i}$ are potentially multisets of size $\left.k_{i}\right)$. Then

$$
\left|e\left(S_{1}, \ldots, S_{t}\right)-\frac{k !|E(H)|}{n^{k}} \prod_{i=1}^{t}\right| S_{i}|| \leqslant \lambda_{2, \pi}(H) \sqrt{\left|S_{1}\right| \cdots\left|S_{t}\right|},
$$

where $e\left(S_{1}, \ldots, S_{t}\right)$ is the number of ordered tuples $\left(s_{1}, \ldots, s_{t}\right)$ such that $s_{1} \cup$ $\cdots \cup s_{t} \in E(H)$ and $s_{i} \in S_{i}$.

Proof. Let $q=k !|E(H)| / n^{k}$, let $\tau_{H}$ be the adjacency map of $H$, and let $\sigma=\tau_{H}-$ $q J$. It is easy to see that, by definition, $(\tau-q J)_{\vec{\pi}}=\tau_{\vec{\pi}}-q J_{\vec{\pi}}$, so $\lambda_{2, \pi}(H)=\left\|\sigma_{\vec{\pi}}\right\|$. 
Let $\chi_{S_{i}} \in W^{k_{i} \otimes}$ be the indicator tensor of $S_{i}$. If we let $V(H)=[n]$, then

$$
\chi_{S_{i}}=\sum_{\substack{\left\{v_{1}, \ldots, v_{k_{i}}\right\} \in S_{i} \\ v_{1} \leqslant \cdots \leqslant v_{k_{i}}}}\left(e_{v_{1}} \otimes \cdots \otimes e_{v_{k_{i}}}\right) .
$$

By the linearity of $\sigma_{\vec{\pi}}$ and the definition of $J_{\vec{\pi}}$,

$$
\begin{aligned}
\sigma_{\vec{\pi}}\left(\chi_{S_{1}}, \ldots, \chi_{S_{t}}\right) & =\tau_{\vec{\pi}}\left(\chi_{S_{1}}, \ldots, \chi_{S_{t}}\right)-q J_{\vec{\pi}}\left(\chi_{S_{1}}, \ldots, \chi_{S_{t}}\right) \\
& =e\left(S_{1}, \ldots, S_{t}\right)-q \prod_{i=1}^{t}\left|S_{i}\right| .
\end{aligned}
$$

Before upper bounding this by $\lambda_{2, \pi}(H)$, we must scale each indicator tensor to be unit length. Since $\left\{e_{j_{1}} \otimes \cdots \otimes e_{j_{k_{i}}}: 1 \leqslant j_{1}, \ldots, j_{k_{i}} \leqslant n\right\}$ forms a basis of $W^{\otimes k_{i}}$, we have $\left\|\chi_{S_{i}}\right\|=\sqrt{\left|S_{i}\right|}$. Thus

$$
\left|\sigma_{\vec{\pi}}\left(\frac{\chi_{S_{1}}}{\left\|\chi_{S_{1}}\right\|}, \ldots, \frac{\chi_{S_{t}}}{\left\|\chi_{S_{t}}\right\|}\right)\right| \leqslant\left\|\sigma_{\vec{\pi}}\right\|=\lambda_{2, \pi}(H) .
$$

Consequently,

$$
\left|\sigma_{\vec{\pi}}\left(\chi_{S_{1}}, \ldots, \chi_{S_{t}}\right)\right| \leqslant \lambda_{2, \pi}(H)\left\|\chi_{S_{1}}\right\| \cdots\left\|\chi_{S_{t}}\right\|=\lambda_{2, \pi}(H) \sqrt{\left|S_{1}\right| \cdots\left|S_{t}\right|},
$$

and the proof is complete.

LEMMA 4. Let $\mathcal{H}=\left\{H_{n}\right\}$ be a sequence of $k$-uniform hypergraphs with loops with $\left|V\left(H_{n}\right)\right|=n$ and $\left|E\left(H_{n}\right)\right| \geqslant p\left(\begin{array}{l}n \\ k\end{array}\right)+o\left(n^{k}\right)$. Let $\tau_{n}$ be the adjacency map of $H_{n}$, and let $\vec{\pi}=\left(k_{1}, \ldots, k_{t}\right)$ be a proper ordered partition of $k$. If $\lambda_{1, \pi}\left(H_{n}\right)=$ $p n^{k / 2}+o\left(n^{k / 2}\right)$, then $\left|E\left(H_{n}\right)\right|=p\left(\begin{array}{l}n \\ k\end{array}\right)+o\left(n^{k}\right)$.

Proof. Throughout this proof, the subscripts on $n$ are dropped for simplicity. Let $W$ be the vector space over $\mathbb{R}$ of dimension $n$. For $1 \leqslant i \leqslant t$, let $\overrightarrow{1}_{k_{i}}$ denote the all-ones vector in $W^{\otimes k_{i}}$, so $\left\|\overrightarrow{1}_{k_{i}}\right\|=n^{k_{i} / 2}$. Then

$$
\begin{aligned}
\tau_{\vec{\pi}}\left(\frac{\overrightarrow{1}_{k_{1}}}{n^{k_{1} / 2}}, \ldots, \frac{\overrightarrow{1}_{k_{t}}}{n^{k_{t} / 2}}\right) & =\frac{1}{n^{k / 2}} \tau_{\vec{\pi}}\left(\overrightarrow{1}_{k_{1}}, \ldots, \overrightarrow{1}_{k_{t}}\right)=\frac{1}{n^{k / 2}} \tau\left(\overrightarrow{1}_{1}, \ldots, \overrightarrow{1}_{1}\right) \\
& =\frac{1}{n^{k / 2}} \sum_{i_{1}, \ldots, i_{k}=1}^{n} \tau\left(e_{i_{1}}, \ldots, e_{i_{k}}\right) \\
& =\frac{1}{n^{k / 2}} k !|E(H)| .
\end{aligned}
$$


Thus the spectral norm of $\tau_{\vec{\pi}}$ is at least $k !|E(H)| / n^{k / 2}$, so

$$
p n^{k / 2} \leqslant \frac{k !|E(H)|}{n^{k / 2}}+o\left(n^{k / 2}\right) \leqslant\left\|\tau_{\vec{\pi}}\right\|+o\left(n^{k / 2}\right)=p n^{k / 2}+o\left(n^{k / 2}\right) .
$$

This implies equality (up to $o\left(n^{k / 2}\right)$ ) throughout the above expression. In particular, $\left|E\left(H_{n}\right)\right|=p\left(\begin{array}{l}n \\ k\end{array}\right)+o\left(n^{k}\right)$.

Proof that $\operatorname{Eig}[\pi] \Rightarrow \operatorname{Expand}[\pi]$. First, $\operatorname{Eig}[\pi]$ contains the assertion that $\lambda_{1, \pi}\left(H_{n}\right)=p n^{k / 2}+o\left(n^{k / 2}\right)$, which by Lemma 4 implies that $\left|E\left(H_{n}\right)\right|=p\left(\begin{array}{l}n \\ k\end{array}\right)+$ $o\left(n^{k}\right)$. Consequently, $k !\left|E\left(H_{n}\right)\right| / n^{k}=(1+o(1)) p$ and Theorem 3 imply that

$$
\left|e\left(S_{1}, \ldots, S_{t}\right)-(1+o(1)) p \prod_{i=1}^{t}\right| S_{i}|| \leqslant \lambda_{2, \pi}(H) \sqrt{\left|S_{1}\right| \cdots\left|S_{t}\right|}
$$

for any choice of $S_{i} \subseteq\left(\begin{array}{c}V\left(H_{n}\right) \\ k_{i}\end{array}\right), i=1, \ldots, t$. Since $\pi$ is a partition of $k$, $\sqrt{\left|S_{1}\right| \cdots\left|S_{t}\right|}=O\left(n^{k / 2}\right)$. Also, Eig[ $[\pi]$ states that $\lambda_{2, \pi}(H)=o\left(n^{k / 2}\right)$. Thus (2) becomes

$$
\left|e\left(S_{1}, \ldots, S_{t}\right)-p\right| S_{1}|\cdots| S_{t}||=o\left(n^{k}\right),
$$

which proves Expand $[\pi]$.

\section{5. $\operatorname{Expand}[\pi] \Rightarrow$ Count $[\pi$-linear $]$}

The proof that Expand $[\pi] \Rightarrow$ Count $[\pi$-linear] follows from an embedding lemma for hypergraphs. The proof of Proposition 5 below is a generalization of an argument by Kohayakawa et al. [28], who proved it in the special case of linear hypergraphs, so we omit the proof. A detailed proof appears online [31]. The proposition below is stated for ordered partitions $\vec{\pi}$, but it is easy to see that the proposition is independent of the ordering chosen for $\vec{\pi}$.

PROPOSITION 5. Let $\vec{\pi}=\left(k_{1}, \ldots, k_{t}\right)$ be a proper ordered partition of $k$, let $0<p<1$, and let $F$ be any fixed $k$-uniform, $\pi$-linear hypergraph with $f$ vertices and $m$ edges.

Let $\mathcal{H}=\left\{H_{n}\right\}_{n \rightarrow \infty}$ be a sequence of $k$-uniform hypergraphs with loops with $\left|V\left(H_{n}\right)\right|=n,\left|E\left(H_{n}\right)\right|=p\left(\begin{array}{l}n \\ k\end{array}\right)+o\left(n^{k}\right)$, and for which Expand[ $\left.\vec{\pi}\right]$ holds. In other words, for every $S_{1} \subseteq\left(\begin{array}{c}V(H) \\ k_{1}\end{array}\right), \ldots, S_{t} \subseteq\left(\begin{array}{c}V(H) \\ k_{t}\end{array}\right)$, we have $e\left(S_{1}, \ldots, S_{t}\right)=$ $p\left|S_{1}\right| \cdots\left|S_{t}\right|+o\left(n^{k}\right)$. Then the number of labeled copies of $F$ in $H$ is $p^{m} n^{f}+$ $o\left(n^{f}\right)$. 


\section{6. $\mathrm{CyCle}_{4 \ell}[\pi] \Rightarrow \operatorname{Eig}[\pi]$}

In this section, we prove that, if $\mathcal{H}$ is a sequence of $d$-coregular, $k$-uniform hypergraphs with loops which satisfies $\mathrm{CyCl}_{4 \ell}[\pi]$, then $\mathcal{H}$ satisfies $\operatorname{Eig}[\pi]$. Indeed, if $H$ is $d$-coregular with loops, then $\lambda_{1, \pi}(H)=d n^{k / 2-1}$, and the vectors maximizing $\tau_{\vec{\pi}}$ are the all-ones vectors scaled to unit length (see [21, 22]). These facts simplify the proof of $\mathrm{CyCl}_{4 \ell}[\pi] \Rightarrow \mathrm{Eig}[\pi]$ which appears in this section. In a companion paper [32], we develop the additional algebra required to prove $\mathrm{CyCl}_{4 \ell}[\pi] \Rightarrow \mathrm{Eig}[\pi]$ for all sequences. Throughout this section, let $0<p<1$ be a fixed integer, and define $d=d(n)=\lfloor p n\rfloor$.

First, let us recall the proof of $\mathrm{CyCle}_{4}[1+1] \Rightarrow \mathrm{Eig}[1+1]$ for graphs. Let $A$ be the adjacency matrix of a $d$-regular graph $G$. Then $\operatorname{Tr}\left[A^{4}\right]$ is the number of circuits of length 4 , so Cycle $e_{4}[1+1]$ implies that $\operatorname{Tr}\left[A^{4}\right]=d^{4}+o\left(n^{4}\right)$. Since $G$ is $d$-regular, the largest eigenvalue of $A^{4}$ is $d^{4}$, so all eigenvalues of $A$ besides $d$ are $o(n)$ in absolute value, completing the proof that $\mathrm{Eig}[1+1]$ holds. Our proof for hypergraphs follows the same outline once some algebraic facts about multilinear maps are proved. In Section 6.1, we define (nonstandard) products and powers of multilinear maps. In Section 6.2, we show that the powers of multilinear maps count walks and that the trace of the powers of multilinear maps counts circuits. Finally, Section 6.3 contains the proof that $\mathrm{Cycle}_{4 \ell}[\pi] \Rightarrow \mathrm{Eig}[\pi]$.

6.1. Products and powers of multilinear maps. In this section, we give (nonstandard) definitions of the products and powers of multilinear maps.

DEFINITION. Let $V_{1}, \ldots, V_{t}$ be finite-dimensional vector spaces over $\mathbb{R}$, and let $\phi, \psi: V_{1} \times \cdots \times V_{t} \rightarrow \mathbb{R}$ be $t$-linear maps. The product of $\phi$ and $\psi$, written $\phi * \psi$, is a $(t-1)$-linear map defined as follows. Let $u_{1}, \ldots, u_{t-1}$ be vectors, where $u_{i} \in V_{i}$. Let $\left\{b_{1}, \ldots, b_{\operatorname{dim}\left(V_{t}\right)}\right\}$ be any orthonormal basis of $V_{t}$.

$$
\begin{gathered}
\phi * \psi:\left(V_{1} \otimes V_{1}\right) \times\left(V_{2} \otimes V_{2}\right) \times \cdots \times\left(V_{t-1} \otimes V_{t-1}\right) \rightarrow \mathbb{R} \\
\phi * \psi\left(u_{1} \otimes v_{1}, \ldots, u_{t-1} \otimes v_{t-1}\right):=\sum_{j=1}^{\operatorname{dim}\left(V_{t}\right)} \phi\left(u_{1}, \ldots, u_{t-1}, b_{j}\right) \psi\left(v_{1}, \ldots, v_{t-1}, b_{j}\right) .
\end{gathered}
$$

Extend the map $\phi * \psi$ linearly to all of the domain to produce a $(t-1)$-linear map.

It is straightforward to see that the above definition is well defined: the map is the same for any choice of orthonormal basis by the linearity of $\phi$ and $\psi$. A proof of this fact appears in [32]. 
Definition. Let $V_{1}, \ldots, V_{t}$ be finite-dimensional vector spaces over $\mathbb{R}$, let $\phi$ : $V_{1} \times \cdots \times V_{t} \rightarrow \mathbb{R}$ be a $t$-linear map, and let $s$ be an integer $0 \leqslant s \leqslant t-1$. Define

$$
\phi^{2^{s}}: V_{1}^{\otimes 2^{s}} \times \cdots \times V_{t-s}^{\otimes 2^{s}} \rightarrow \mathbb{R},
$$

where $\phi^{2^{0}}:=\phi$ and $\phi^{2^{s}}:=\phi^{2^{s-1}} * \phi^{2^{s-1}}$.

Note that we only define this for exponents which are powers of two, because the product $*$ is only defined when the domains of the maps are the same. An expression like $\phi^{3}=\phi *(\phi * \phi)$ does not make sense, because $\phi$ and $\phi * \phi$ have different domains. This defines the power $\phi^{2^{t-1}}$, which is a linear map $V_{1}^{\otimes 2^{t-1}} \rightarrow$ $\mathbb{R}$.

DEFINITION. Let $V_{1}, \ldots, V_{t}$ be finite-dimensional vector spaces over $\mathbb{R}$, let $\phi$ : $V_{1} \times \cdots \times V_{t} \rightarrow \mathbb{R}$ be a $t$-linear map, and define $A\left[\phi^{2^{t-1}}\right]$ to be the following square matrix/bilinear map. Let $u_{1}, \ldots, u_{2^{t-2}}, v_{1}, \ldots, v_{2^{t-2}}$ be vectors, where $u_{i}, v_{i} \in V_{1}$.

$$
\begin{gathered}
A\left[\phi^{2^{t-1}}\right]: V_{1}^{\otimes 2^{t-2}} \times V_{1}^{\otimes 2^{t-2}} \rightarrow \mathbb{R} \\
A\left[\phi^{2^{t-1}}\right]\left(u_{1} \otimes \cdots \otimes u_{2^{t-2}}, v_{1} \otimes \cdots v_{2^{t-2}}\right) \\
:=\phi^{2^{t-1}}\left(u_{1} \otimes v_{1} \otimes u_{2} \otimes v_{2} \otimes \cdots \otimes u_{2^{t-2}} \otimes v_{2^{t-2}}\right) .
\end{gathered}
$$

Extend the map linearly to the entire domain to produce a bilinear map.

It is straightforward to check that, by definition, $A\left[\phi^{2^{t-1}}\right]$ is a square symmetric real-valued matrix for any $\phi$; a proof of this fact appears in [32].

6.2. Counting walks and circuits. This section contains the proof of the following proposition.

PROPOSITION 6. Let $H$ be a $k$-uniform hypergraph with loops, let $\vec{\pi}$ be a proper ordered partition of $k$, and let $\ell \geqslant 2$ be an integer. Let $\tau$ be the adjacency map of $H$. Then $\operatorname{Tr}\left[A\left[\tau_{\vec{\pi}}^{2^{t-1}}\right]^{\ell}\right]$ is the number of labeled circuits of type $\vec{\pi}$ and length $2 \ell$ in $H$.

The proof of this proposition comes down to showing that the function $\tau_{\vec{\pi}}^{2^{t-1}}$ counts the step $S_{\vec{\pi}}$. We do this by induction by describing exactly the hypergraph counted by $\tau_{\vec{\pi}}^{2^{s}}$, which is the following hypergraph.

Definition. For $\vec{\pi}=(1, \ldots, 1)$ with $t$ parts, let $0 \leqslant s \leqslant t-1$, and define the hypergraph $D_{\vec{\pi}, s}$ as follows. Let $A_{1}, \ldots, A_{t-s}$ be disjoint sets of size $2^{s}$ where 
elements are labeled by binary strings of length $s$, and let $B_{t-s+1}, \ldots, B_{t}$ be disjoint sets of size $2^{s-1}$ where elements are labeled by binary strings of length $s-1$. The vertex set of $D_{\vec{\pi}, s}$ is $A_{1} \dot{\cup} \ldots \dot{\cup} A_{t-s} \dot{\cup} B_{t-s+1} \dot{\cup} \ldots \dot{\cup} B_{t}$. Make $a_{1}, \ldots$, $a_{t-s}, b_{t-s+1}, \ldots, b_{t}$ an edge of $D_{\vec{\pi}, s}$ if $a_{i} \in A_{i}, b_{j} \in B_{j}$, the codes for $a_{1}, \ldots, a_{t-s}$ are all equal, and the code for $b_{t-s+j}$ is equal to the code formed by removing the $j$ th bit of the code for $a_{1}$.

For a general $\vec{\pi}=\left(k_{1}, \ldots, k_{t}\right)$, start with $D_{(1, \ldots, 1), s}$ and expand each vertex into the appropriate size; that is, a vertex in $A_{i}$ is expanded into $k_{i}$ vertices, and each vertex in $B_{j}$ is expanded into $k_{j}$ vertices. In $D_{\vec{\pi}, s}$, each vertex in $A_{i}$ is labeled by a pair $(c, z)$, where $c$ is a bit string of length $s$ and $z \in\left[k_{i}\right]$. We call $z$ the expansion index of the vertex.

The hypergraph $D_{\vec{\pi}, 0}$ is a single edge, and the hypergraph $D_{\vec{\pi}, t-1}$ is by definition the step $S_{\vec{\pi}}$. The following lemma precisely formulates what we mean when we say that $\tau_{\vec{\pi}}^{2^{s}}$ counts the hypergraph $D_{\vec{\pi}, s}$.

LEMMA 7. Let $H$ be a $k$-uniform hypergraph with loops, let $\vec{\pi}$ be a proper ordered partition of $k$ with $\vec{\pi}=\left(k_{1}, \ldots, k_{t}\right)$, and let $0 \leqslant s \leqslant t-1$. Let $W$ be the vector space over $\mathbb{R}$ of dimension $|V(H)|$, and let $\tau$ be the adjacency map of $H$. Let $A_{1}, \ldots, A_{t-s}, B_{t-s+1}, \ldots, B_{t}$ be the vertex sets in the definition of $D_{\vec{\pi}, s}$, and let $\Delta$ be any map $A_{1} \cup \cdots \cup A_{t-s} \rightarrow V(H)$. Then $\tau_{\vec{\pi}}^{s}$ counts the number of labeled, possibly degenerate copies of $D_{\vec{\pi}, s}$ extending $\Delta$ as follows.

Let $a_{i, 1}, \ldots, a_{i, k_{i} 2^{s}}$ be the vertices of $A_{i}$ ordered first lexicographically by bit code and then for equal codes ordered by expansion index. Let $\chi_{i}$ be the indicator tensor in $W^{\otimes k_{i} 2^{s}}$ for the vertex tuple $\left(\Delta\left(a_{i, 1}\right), \ldots, \Delta\left(a_{i, k_{i} 2^{s}}\right)\right)$. Then $\tau_{\vec{\pi}}^{2^{s}}\left(\chi_{1}, \ldots\right.$, $\left.\chi_{t-s}\right)$ is the number of edge-preserving maps $V\left(D_{\vec{\pi}, s}\right) \rightarrow V(H)$ which are consistent with $\Delta$.

Proof. The proof is done by induction on $s$. The base case is $s=0$, where $D_{\vec{\pi}, 0}$ is a single edge, there are no $B$-type sets, and thus $\Delta$ is a map $V\left(D_{\vec{\pi}, 0}\right) \rightarrow V(H)$. The number of edge-preserving maps extending $\Delta$ is either zero or one depending on if the image of $\Delta$ is an edge of $H$ or not. But $\tau_{\vec{\pi}}\left(\chi_{1}, \ldots, \chi_{t}\right)$ equals zero or one depending on if the vertices defining the indicator tensors $\chi_{i}$ form an edge, exactly what is required.

Assume that the lemma is true for $s$; we will prove it for $s+1$. Denote by $\hat{A}_{1}, \ldots, \hat{A}_{t-s-1}, \hat{B}_{t-s}, \ldots, \hat{B}_{t}$ the sets in the definition of $D_{\vec{\pi}, s+1}$ and by $A_{1}, \ldots$, $A_{t-s}, B_{t-s+1}, \ldots, B_{t}$ the sets in the definition of $D_{\vec{\pi}, s}$. Let $\hat{\Delta}$ be a map $\hat{A}_{1} \cup \cdots \cup$ $\hat{A}_{t-s-1} \rightarrow V(H)$, and let $\hat{\chi}_{1}, \ldots, \hat{\chi}_{t-s-1}$ be the indicator tensors for the image of $\hat{\Delta}$ ordered as in the statement of the lemma. Since $\hat{\chi}_{i}$ is an indicator tensor in $W^{\otimes k_{i} 2^{s+1}}$, it is a simple tensor, so $\hat{\chi}_{i}=\chi_{i} \otimes \chi_{i}^{\prime}$ for $\chi_{i}, \chi_{i}^{\prime} \in W^{\otimes k_{i} 2^{s}}$. Note that 


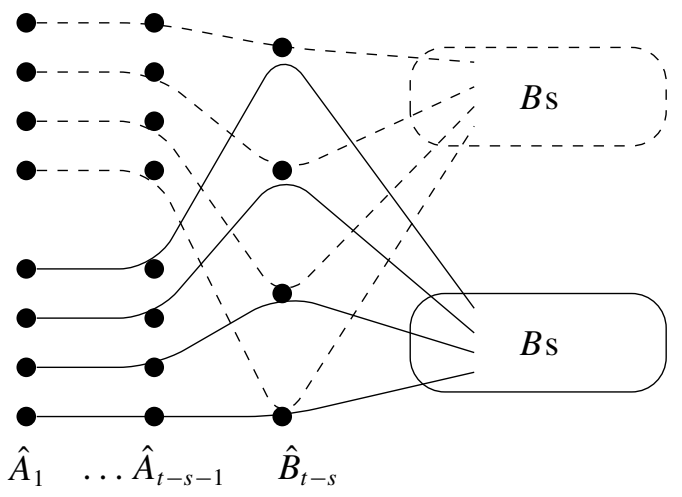

(a) $D_{\vec{\pi}, s+1}$

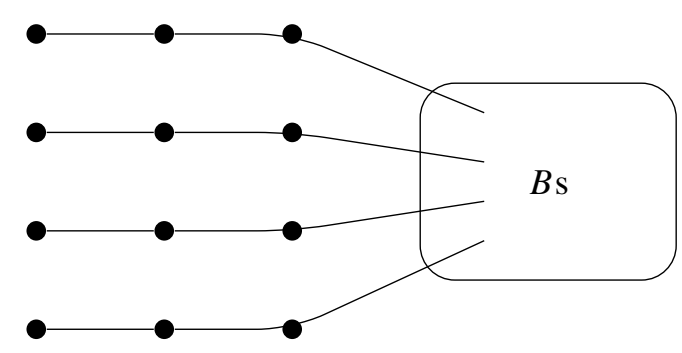

$A_{1} \quad \ldots A_{t-s-1} \quad A_{t-s}$

(b) $D_{\vec{\pi}, s}$

$$
\begin{aligned}
\tau_{\vec{\pi}}^{2^{s+1}} & \left(\chi_{1} \otimes \chi_{1}^{\prime}, \ldots, \chi_{t-s-1} \otimes \chi_{t-s-1}^{\prime}\right) \\
& =\sum_{j=1}^{d} \tau_{\vec{\pi}}^{2^{s}}\left(\chi_{1}, \ldots, \chi_{t-s-1}, w_{j}\right) \tau_{\vec{\pi}}^{2^{s}}\left(\chi_{1}^{\prime}, \ldots, \chi_{t-s-1}^{\prime}, w_{j}\right)
\end{aligned}
$$

Figure 5: The induction step of Lemma 7.

$\chi_{i}$ is the indicator tensor for the image under $\hat{\Delta}$ of the vertices of $D_{\vec{\pi}, s+1}$ whose code starts with zero, and $\chi_{i}^{\prime}$ is the indicator tensor for the image under $\hat{\Delta}$ of the vertices whose code starts with a one, since the definition of $\hat{\chi}_{i}$ sorted the vertices in the image lexicographically.

Consider the expansion of the definition of $\tau_{\vec{\pi}}^{2^{s+1}}\left(\hat{\chi}_{1}, \ldots, \hat{\chi}_{t-s-1}\right)$ shown in (3) in Figure 5; the tensors $\hat{\chi}_{i}$ are split into $\chi_{i}$ and $\chi_{i}^{\prime}$, and we sum over the standard 
basis $\left\{w_{1}, \ldots, w_{d}\right\}$ of $W^{\otimes k_{t-s} 2^{s}}$, where $d=\operatorname{dim}\left(W^{\otimes k_{t-s} 2^{s}}\right)$. We can consider the tensor $w_{j}$ in (3) to be the indicator tensor of a tuple of $k_{t-s} 2^{s}$ vertices.

Definition. We now describe two embeddings of $D_{\vec{\pi}, s}$ into $D_{\vec{\pi}, s+1}$. In Figure 5(a), these two embeddings are the dotted and solid lines. Let $\Gamma_{0}: V\left(D_{\vec{\pi}, s}\right) \rightarrow V\left(D_{\vec{\pi}, s+1}\right)$ be the following injection. For $1 \leqslant i \leqslant t-s-1$ and $a \in A_{i}$, set $\Gamma_{0}(a)$ equal to the vertex in $\hat{A}_{i}$ whose code equals the code for $a$ with a zero prepended to the code and the same expansion index. That is, a vertex in $A_{i}$ with label $(1011,4)$ is mapped to the vertex in $\hat{A}_{i}$ with label $(01011,4)$. For $a \in A_{t-s}$, set $\Gamma_{0}(a)$ equal to the vertex in $\hat{B}_{t-s}$ which has the same label as $a$. For $t-s+1 \leqslant j \leqslant t$ and $b \in B_{j}$, set $\Gamma_{0}(b)$ equal to the vertex in $\hat{B}_{j}$ whose code equals the code for $b$ with a zero prepended to the code and the same expansion index. In other words, $\Gamma_{0}$ adds a zero to the front of the codes except for vertices in $A_{t-s}$ whose code does not change. Define $\Gamma_{1}: V\left(D_{\vec{\pi}, s}\right) \rightarrow V\left(D_{\vec{\pi}, s+1}\right)$ similarly, except prepend a one instead of a zero. In Figure 5(a), the dotted lines represent $\Gamma_{0}$ and the solid lines represent $\Gamma_{1}$.

Claim. $\Gamma_{0}$ and $\Gamma_{1}$ are edge-preserving injections, and every edge in $D_{\vec{\pi}, s+1}$ is in the image of $\Gamma_{0}$ or $\Gamma_{1}$ but not both.

Proof of Claim. Let $E$ be an edge in $D_{\vec{\pi}, s}$. For $1 \leqslant i \leqslant j \leqslant t-s-1$ and $a_{i} \in A_{i} \cap E$ and $a_{j} \in A_{j} \cap E$, since $E$ is an edge of $D_{\vec{\pi}, s}$, the code for $a_{i}$ equals the code for $a_{j}$. This implies that the codes for $\Gamma_{0}\left(a_{i}\right)$ and $\Gamma_{0}\left(a_{j}\right)$ are equal, since both had a zero prepended. Now consider $b \in A_{t-s} \cap E$ which is mapped to $\hat{B}_{t-s}$. The conditions for $\Gamma_{0}(E)$ to be an edge of $D_{\vec{\pi}, s+1}$ requires that the code for $\Gamma_{0}(b)$ equals the code formed by deleting the first bit of $\Gamma_{0}(a)$, where $a \in A_{1} \cap E$. But the code for $a$ equals the code for $b$, since both are in $A$-type sets in $D_{\vec{\pi}, s}$, and the map $\Gamma_{0}$ adds a zero to the front of the code for $a$ and leaves the code for $b$ alone. Thus the code for $\Gamma_{0}(b)$ equals the code formed by deleting the first bit of $\Gamma_{0}(a)$. Lastly, consider $b \in B_{j} \cap E$ for $t-s+1 \leqslant j \leqslant t$, and consider deleting the $(j+1)$ th bit of the code for $\Gamma_{0}(a)$. This is the same as deleting the $j$ th bit of $a$, since $\Gamma_{0}(a)$ had a zero prepended. But deleting the $j$ th bit of $a$ equals the code for $b$, since $a, b \in E \in E\left(D_{\vec{\pi}, s}\right)$. Thus deleting the $(j+1)$ th bit of $\Gamma_{0}(a)$ gives the code for $\Gamma_{0}(b)$. We have now checked all the conditions, so $\Gamma_{0}(E)$ is an edge of $D_{\vec{\pi}, s+1}$; that is, $\Gamma_{0}$ is edge preserving. $\Gamma_{1}$ is edge preserving by the same argument. Finally, let $E$ be an edge of $D_{\vec{\pi}, s+1}$, and pick $a \in E \cap \hat{A}_{1}$. If the first bit of the code for $a$ equals zero, then $E$ is in the image of $\Gamma_{0}$, and if the first bit of the code for $a$ equals one, then $E$ is in the image of $\Gamma_{1}$. This concludes the proof of the claim.

This claim implies that any edge-preserving map extending $\hat{\Delta}$ is formed from two edge-preserving maps $V\left(D_{\vec{\pi}, s}\right) \rightarrow V(H)$ each extending the appropriate 
restriction of $\hat{\Delta}$. Start with $\hat{\Delta}$, and extend arbitrarily to a map $\Lambda: \hat{A}_{1} \cup \cdots \cup$ $\hat{A}_{t-s-1} \cup \hat{B}_{t-s} \rightarrow V(H)$. Next define $\Lambda_{0}$ and $\Lambda_{1}$ as maps $A_{1} \cup \cdots \cup A_{t-s} \rightarrow V(H)$ such that $\Lambda_{0}=\left.\Lambda \circ \Gamma_{0}\right|_{\bar{A}}$ and $\Lambda_{1}=\left.\Lambda \circ \Gamma_{1}\right|_{\bar{A}}$, where $\bar{A}=A_{1} \cup \cdots \cup A_{t-s}$, so $\left.\Gamma_{0}\right|_{\bar{A}}$ is the map $\Gamma_{0}$ restricted to the $A$-type sets in $D_{\vec{\pi}, s}$. By the claim, the number of edge-preserving maps extending $\hat{\Delta}$ equals the sum over $\Lambda$ of the product of the number of edge-preserving maps extending $\Lambda_{0}$ and extending $\Lambda_{1}$. This is because any edge-preserving map extending $\Lambda$ can be composed with $\Gamma_{0}$ and $\Gamma_{1}$ to create edge-preserving maps extending $\Lambda_{0}$ and $\Lambda_{1}$, and, since $\Gamma_{0}$ and $\Gamma_{1}$ are injections covering all edges of $D_{\vec{\pi}, s+1}$, this can be reversed. The last step in the proof is to show that this is exactly what (3) counts.

Let $\hat{b}_{1}, \ldots, \hat{b}_{k_{t-s} 2^{s}}$ be the vertices of $\hat{B}_{t-s}$ listed first in lexicographic order of codes and then by expansion index. Let $w$ be the indicator tensor in $W^{\otimes k_{t-s} 2^{s}}$ for the vertex tuple $\left(\Lambda\left(\hat{b}_{1}\right), \ldots, \Lambda\left(\hat{b}_{k_{t-s} 2^{s}}\right)\right)$. Note that, as $\Lambda$ ranges over all possible extensions of $\hat{\Delta}, w$ ranges over the standard basis of $W^{\otimes k_{t-s} 2^{s}}$. Now $\chi_{1}, \ldots$, $\chi_{t-s-1}, w$ are the indicator tensors representing the image of the map $\Lambda_{0}$, since, as mentioned above, $\chi_{1}, \ldots, \chi_{t-s-1}$ are the indicator tensors for the image under $\hat{\Delta}$ of the vertices whose code stars with a zero. Similarly, $\chi_{1}^{\prime}, \ldots, \chi_{t-s-1}^{\prime}, w$ are the indicator tensors representing the image of the map $\Lambda_{1}$. Thus, by induction, $\tau_{\vec{\pi}}^{2^{s}}\left(\chi_{1}, \ldots, \chi_{t-s-1}, w\right)$ is the number of edge-preserving maps extending $\Lambda_{0}$, and $\tau_{\vec{\pi}}^{2^{s}}\left(\chi_{1}^{\prime}, \ldots, \chi_{t-s-1}^{\prime}, w\right)$ is the number of edge-preserving maps extending $\Lambda_{1}$. By the claim, this implies that the product

$$
\tau_{\overrightarrow{\tilde{\pi}}}^{2^{s}}\left(\chi_{1}, \ldots, \chi_{t-s-1}, w\right) \tau_{\vec{\pi}}^{2^{s}}\left(\chi_{1}^{\prime}, \ldots, \chi_{t-s-1}^{\prime}, w\right)
$$

counts the number of edge-preserving maps extending $\Lambda$. Thus (3) sums over the choices for $\Lambda$ extending $\hat{\Delta}$ of the number of edge-preserving maps extending $\Lambda$. This sum is exactly the number of edge-preserving maps extending $\hat{\Delta}$, so the proof is complete.

COROLLARY 8. Let $H$ be a $k$-uniform hypergraph with loops, let $\vec{\pi}$ be a proper ordered partition of $k$ with $\vec{\pi}=\left(k_{1}, \ldots, k_{t}\right)$, and let $\ell \geqslant 2$ be an integer. Let $W$ be the vector space over $\mathbb{R}$ of dimension $|V(H)|$, and let $\tau$ be the adjacency map of $H$. Let $a_{1}, \ldots, a_{k_{1} 2^{t-2}}, a_{1}^{\prime}, \ldots, a_{k_{1} 2^{t-2}}^{\prime}$ be (not necessarily distinct) vertices of $H$, and let $\xi$ and $\xi^{\prime}$ be the indicator tensors in $W^{k_{1} 2^{t-2}}$ for the tuples $\left(a_{1}, \ldots, a_{k_{1} 2^{t-2}}\right)$ and $\left(a_{1}^{\prime}, \ldots, a_{k_{1} 2^{t-2}}^{\prime}\right)$, respectively. Then $A\left[\tau_{\vec{\pi}}^{2^{t-1}}\right]\left(\xi, \xi^{\prime}\right)$ is the number of labeled, possibly degenerate steps of type $\vec{\pi}$ in $H$ with attach tuples $\left(a_{1}, \ldots, a_{k_{1} 2^{t-2}}\right)$ and $\left(a_{1}^{\prime}, \ldots, a_{k_{1} t^{t-2}}^{\prime}\right)$. Also, $A\left[\tau_{\vec{\pi}}^{2^{t-1}}\right]^{\ell}\left(\xi, \xi^{\prime}\right)$ is the number of labeled walks of length $2 \ell$ and type $\vec{\pi}$ with attach tuples $\left(a_{1}, \ldots, a_{k_{1} 2^{t-2}}\right)$ and $\left(a_{1}^{\prime}, \ldots, a_{k_{1} 2^{t-2}}^{\prime}\right)$.

Proof. The proof is by induction on $\ell$. First, consider the base case of $\ell=1$, where the path of length two and type $\vec{\pi}$ is the step of type $\vec{\pi}$. Let $A$ be the 
vertex set from the definition of the step $S_{\vec{\pi}}$. Define a mapping $\Delta: A \rightarrow V(H)$ by mapping the attach tuples of $S_{\vec{\pi}}$ to the tuples $\left(a_{1}, \ldots, a_{k_{1} 2^{t-2}}\right)$ and $\left(a_{1}^{\prime}, \ldots\right.$, $\left.a_{k_{1} 2^{t-2}}^{\prime}\right)$ in $V(H)$. By definition, the first attach tuple of $S_{\vec{\pi}}$ is the vertices ending with a zero and listed in lexicographic order, and the second attach tuple of $S_{\vec{\pi}}$ is the vertices ending with a one and listed in lexicographic order. This implies that the indicator tensor $\chi_{1}$ from the statement of Lemma 7 is the indicator tensor in $W^{\otimes k_{1} 2^{t-1}}$ for the tuple $\left(a_{1}, \ldots, a_{k_{1}}, a_{1}^{\prime}, \ldots, a_{k_{1}}^{\prime}, a_{k_{1}+1}, \ldots, a_{2 k_{1}}, a_{k_{1}+1}^{\prime}\right.$, $\left.\ldots, a_{2 k_{1}}^{\prime}, \ldots, a_{k_{1} 2^{t-3}+1}, \ldots, a_{k_{1} 2^{t-2}}, a_{k_{1} 2^{t-3}+1}^{\prime}, \ldots, a_{k_{1} 2^{t-2}}^{\prime}\right)$, since each attach tuple is in lexicographic order but the last bit is zero or one, so the full ordering alternates between attach tuples. By the definition of $A\left[\tau_{\vec{\pi}}^{2^{t-1}}\right]$ and the indicator tensors $\xi, \xi^{\prime}$, $\chi_{1}, A\left[\tau_{\vec{\pi}}^{2^{t-1}}\right]\left(\xi, \xi^{\prime}\right)=\tau_{\vec{\pi}}^{2^{t-1}}\left(\chi_{1}\right)$. Thus Lemma 7 applied with $s=t-1$ shows that the number of edge-preserving maps extending $\Delta$ is $A\left[\tau_{\vec{\pi}}^{2^{t-1}}\right]\left(\xi, \xi^{\prime}\right)$, but, by the definition of $\Delta$, this is exactly the number of labeled, possibly degenerate steps of type $\vec{\pi}$ with attach tuples $\left(a_{1}, \ldots, a_{k_{1} 2^{t-2}}\right)$ and $\left(a_{1}^{\prime}, \ldots, a_{k_{1} 2^{t-2}}^{\prime}\right)$.

Next assume that the corollary is true for $\ell$; we will show that it is true for $\ell+1$. Using the definition of matrix multiplication, let $\left\{d_{1}, \ldots, d_{\operatorname{dim}\left(W^{\otimes k_{1} 2^{2-2}}\right)}\right\}$ be the standard basis of $W^{\otimes k_{1} 2^{t-2}}$, so

$$
A\left[\tau_{\vec{\pi}}^{2^{t-1}}\right]^{\ell+1}\left(\xi, \xi^{\prime}\right)=\sum_{i=1}^{\operatorname{dim}\left(W^{\otimes k_{1} 2^{t-2}}\right)} A\left[\tau_{\vec{\pi}}^{2^{t-1}}\right]^{\ell}\left(\xi, d_{i}\right) A\left[\tau_{\vec{\pi}}^{2^{t-1}}\right]\left(d_{i}, \xi^{\prime}\right) .
$$

Each standard basis vector $d_{i}$ can be thought of as a $k_{1} 2^{t-2}$-tuple of vertices which corresponds to one of the two attach tuples. Thus (4) sums over the internal attach tuple for a walk of length $2 \ell$ and $S_{\vec{\pi}}$.

Proof of Proposition 6. Since $A\left[\tau_{\vec{\pi}}^{2^{t-1}}\right]^{\ell}$ counts the number of walks of length $2 \ell$, the trace counts circuits. If $\left\{d_{1}, \ldots, d_{\operatorname{dim}\left(W^{\otimes k_{1} 2^{t-2}}\right)}\right\}$ is any orthonormal basis of $W^{\otimes k_{1} 2^{t-2}}$, the trace of the matrix $A\left[\tau_{\vec{\pi}}^{2^{t-1}}\right]^{\ell}$ is

$$
\operatorname{Tr}\left[A\left[\tau_{\vec{\pi}}^{2^{t-1}}\right]^{\ell}\right]=\sum_{i=1}^{\operatorname{dim}\left(W^{\otimes k_{1} 2^{t-2}}\right)} A\left[\tau_{\vec{\pi}}^{2^{t-1}}\right]^{\ell}\left(d_{i}, d_{i}\right) .
$$

If $\left\{d_{1}, \ldots, d_{\operatorname{dim}\left(W^{\left.\otimes k_{1} 2^{t-2}\right)}\right\}}\right.$ is the standard basis, each $d_{i}$ corresponds to a tuple of $k_{1} 2^{t-2}$ vertices, so the above expression is the number of walks of type $\vec{\pi}$ with both attach tuples equal to $d_{i}$.

6.3. Bounding eigenvalues from cycle counts. This section contains the proof that $\mathrm{Cycle}_{4 \ell}[\pi] \Rightarrow \mathrm{Eig}[\pi]$ for $d$-coregular hypergraphs with loops. First, we require a few simple algebraic facts of multilinear maps. 
LEMMA 9. Let $t \geqslant 2$, let $V_{1}, \ldots, V_{t}$ be finite-dimensional vector spaces over $\mathbb{R}$, let $\phi: V_{1} \times \cdots \times V_{t} \rightarrow \mathbb{R}$ be a $t$-linear map, and let $x_{1} \in V_{1}, \ldots, x_{t} \in V_{t}$ be unit length vectors. Then

$$
\left|\phi\left(x_{1}, \ldots, x_{t}\right)\right|^{2} \leqslant\left|\phi^{2}\left(x_{1} \otimes x_{1}, \ldots, x_{t-1} \otimes x_{t-1}\right)\right| .
$$

Proof. Consider the linear map $\phi\left(x_{1}, \ldots, x_{t-1}, \cdot\right)$ which is a linear map from $V_{t}$ to $\mathbb{R}$. There exists a vector $w \in V_{t}$ such that $\phi\left(x_{1}, \ldots, x_{t-1}, \cdot\right)=\langle w, \cdot\rangle$. Then

$$
\begin{aligned}
\phi^{2}\left(x_{1} \otimes x_{1}, \ldots, x_{t-1} \otimes x_{t-1}\right) & =\sum_{j}\left|\phi\left(x_{1}, \ldots, x_{t-1}, b_{j}\right)\right|^{2} \\
& =\sum_{j}\left|\left\langle w, b_{j}\right\rangle\right|^{2}=\langle w, w\rangle,
\end{aligned}
$$

where the last equality is because $\left\{b_{j}\right\}$ is an orthonormal basis of $V_{t}$. Since $\|w\|=$ $\sqrt{\langle w, w\rangle},\left|\phi^{2}\left(x_{1} \otimes x_{1}, \ldots, x_{t-1} \otimes x_{t-1}\right)\right|=|\langle w, w\rangle|=|\langle w, w /\|w\|\rangle|^{2}$. But, since $x_{t}$ is unit length and $\langle w, \cdot\rangle$ is maximized over the unit ball at vectors parallel to $w$ (so maximized at $\pm w /\|w\|$ ), $|\langle w, w /\|w\|\rangle| \geqslant\left|\left\langle w, x_{t}\right\rangle\right|$. Thus

$$
\left|\phi^{2}\left(x_{1} \otimes x_{1}, \ldots, x_{t-1} \otimes x_{t-1}\right)\right|=\mid\left\langle w,\left.\frac{w}{\|w\|}\right|^{2} \geqslant\left|\left\langle w, x_{t}\right\rangle\right|^{2}=\left|\phi\left(x_{1}, \ldots, x_{t}\right)\right|^{2} .\right.
$$

The last equality used the definition of $w$, that $\phi\left(x_{1}, \ldots, x_{t-1}, \cdot\right)=\langle w, \cdot\rangle$.

LEMMA 10. Let $t \geqslant 2$, let $V_{1}, \ldots, V_{t}$ be finite-dimensional vector spaces over $\mathbb{R}$, and let $\phi: V_{1} \times \cdots \times V_{t} \rightarrow \mathbb{R}$ be a t-linear map. Then, for any unit length $x_{1} \in V_{1}, \ldots, x_{t} \in V_{t}$, we have

$$
\left|\phi\left(x_{1}, \ldots, x_{t}\right)\right|^{2^{t-1}} \leqslant\left|A\left[\phi^{2^{t-1}}\right](\underbrace{x_{1} \otimes \cdots \otimes x_{1}}_{2^{t-2}}, \underbrace{x_{1} \otimes \cdots \otimes x_{1}}_{2^{t-2}})\right| .
$$

Also,

$$
\|\phi\|^{2^{t-1}} \leqslant \lambda_{1}\left(A\left[\phi^{2^{t-1}}\right]\right)
$$

Proof. By induction on $s$, we have that

$$
\left|\phi\left(x_{1}, \ldots, x_{t}\right)\right|^{2^{s}} \leqslant\left|\phi^{2^{s}}(\underbrace{x_{1} \otimes \cdots \otimes x_{1}}_{2^{s}}, \ldots, \underbrace{x_{t-s} \otimes \cdots \otimes x_{t-s}}_{2^{s}})\right| .
$$


Indeed, the base case is $s=0$ where both sides are equal and the induction step follows from the previous lemma, since

$$
\begin{aligned}
\left(\left|\phi\left(x_{1}, \ldots, x_{t}\right)\right|^{2^{s-1}}\right)^{2} & \leqslant\left|\phi^{2^{s-1}}(\underbrace{x_{1} \otimes \cdots \otimes x_{1}}_{2^{s-1}}, \ldots, \underbrace{x_{t-s+1} \otimes \cdots \otimes x_{t-s+1}}_{2^{s-1}})\right|^{2} \\
& \leqslant\left|\phi^{2^{s}}(\underbrace{x_{1} \otimes \cdots \otimes x_{1}}_{2^{s}}, \ldots, \underbrace{x_{t-s} \otimes \cdots \otimes x_{t-s}}_{2^{s}})\right|
\end{aligned}
$$

By definition of $A\left[\phi^{2^{t-1}}\right],\left|A\left[\phi^{2^{t-1}}\right]\left(x_{1} \otimes \cdots \otimes x_{1}, x_{1} \otimes \cdots \otimes x_{1}\right)\right|=\mid \phi^{2^{t-1}}\left(x_{1} \otimes \cdots \otimes\right.$ $\left.x_{1}\right) \mid$, completing the proof of (5). Let $x_{1}, \ldots, x_{t}$ be unit length vectors maximizing $\phi$. Since $x_{1} \otimes \cdots \otimes x_{1}$ is unit length, (5) proves that

$$
\begin{aligned}
\|\phi\|^{2^{t-1}}=\left|\phi\left(x_{1}, \ldots, x_{t}\right)\right|^{2^{t-1}} & \leqslant\left|A\left[\phi^{2^{t-1}}\right]\left(x_{1} \otimes \cdots \otimes x_{1}, x_{1} \otimes \cdots \otimes x_{1}\right)\right| \\
& \leqslant \lambda_{1}\left(A\left[\phi^{2^{t-1}}\right]\right) .
\end{aligned}
$$

COROLLARY 11. Let $H$ be a $d$-coregular, $k$-uniform hypergraph with loops, and let $\pi$ be any proper partition of $k$ with $t$ parts. Then, for any ordering $\vec{\pi}$ of $\pi$,

$$
\lambda_{2, \pi}(H) \leqslant\left(\lambda_{2}\left(A\left[\tau_{\vec{\pi}}^{2^{t-1}}\right]\right)\right)^{2^{-t+1}} .
$$

Proof. Let $\vec{\pi}=\left(k_{1}, \ldots, k_{t}\right)$, and let $x_{1}, \ldots, x_{t}$ be unit length vectors maximizing $\tau_{\vec{\pi}}-d / n J_{\vec{\pi}}$ in absolute value, so that $\lambda_{2, \pi}(H)=\left|\left(\tau_{\vec{\pi}}-d / n J_{\vec{\pi}}\right)\left(x_{1}, \ldots, x_{t}\right)\right|$. Write $x_{1}=\alpha y+\beta \hat{1}$, where $y$ is a unit length vector perpendicular to the allones vector, $\hat{1}$ is the all-ones vector scaled to unit length, and $\alpha, \beta \in \mathbb{R}$ with $\alpha^{2}+\beta^{2}=1$. Let $W$ be the vector space over $\mathbb{R}$ of dimension $n$, and for $1 \leqslant i \leqslant t$ let $e_{i, 1}, \ldots, e_{i, n^{k_{i}}}$ be the standard basis of $W^{\otimes k_{i}}$. Since $H$ is $d$-coregular,

$$
\begin{aligned}
\tau_{\vec{\pi}}\left(\hat{1}, x_{2}, \ldots, x_{t}\right)= & \frac{1}{n^{k / 2}} \sum_{1 \leqslant j_{2} \leqslant n^{k_{2}}} \cdots \sum_{1 \leqslant j_{t} \leqslant n^{k_{t}}}\left\langle e_{2, j_{2}}, x_{2}\right\rangle \cdots\left\langle e_{t, j_{t}}, x_{t}\right\rangle \\
& \times \sum_{1 \leqslant j_{1} \leqslant n^{k_{1}}} \tau_{\vec{\pi}}\left(e_{1, j_{1}}, \ldots, e_{t, j_{t}}\right) \\
= & \frac{1}{n^{k / 2}} \sum_{1 \leqslant j_{2} \leqslant n^{k_{2}}} \cdots \sum_{1 \leqslant j_{t} \leqslant n^{k_{t}}}\left\langle e_{2, j_{2}}, x_{2}\right\rangle \cdots\left\langle e_{t, j_{t}}, x_{t}\right\rangle d n^{k_{1}-1} \\
& \times J_{\vec{\pi}}\left(e_{1,1}, e_{2, j_{2}}, \ldots, e_{t, j_{t}}\right) \\
= & \frac{d}{n} J_{\vec{\pi}}\left(\hat{1}, x_{2}, \ldots, x_{t}\right) .
\end{aligned}
$$


Next, $J_{\vec{\pi}}\left(y, x_{2}, \ldots, x_{t}\right)=\langle 1, y\rangle\left\langle 1, x_{2}\right\rangle \cdots\left\langle 1, x_{t}\right\rangle$. Since $y$ is perpendicular to the all-ones vector, $J_{\vec{\pi}}\left(y, x_{2}, \ldots, x_{t}\right)=0$. Therefore, using linearity,

$$
\lambda_{2, \pi}(H)=\left|\left(\tau_{\vec{\pi}}-\frac{d}{n} J_{\vec{\pi}}\right)\left(\alpha y+\beta \hat{1}, x_{2}, \ldots, x_{t}\right)\right|=|\alpha|\left|\tau_{\vec{\pi}}\left(y, x_{2}, \ldots, x_{t}\right)\right| .
$$

By (5) applied to $\tau_{\vec{\pi}}\left(y, x_{2}, \ldots, x_{t}\right), \lambda_{2, \pi}(H) \leqslant|\alpha| \mid A\left[\tau_{\vec{\pi}}^{2^{t-1}}\right](y \otimes \cdots \otimes y, y \otimes$ $\cdots \otimes y)\left.\right|^{2-t+1}$. Since $H$ is $d$-coregular, the number of steps of type $\vec{\pi}$ with a fixed attach tuple $A^{(0)}$ is independent of the choice of $A^{(0)}$. By Corollary 8, each row of the matrix $A\left[\tau_{\vec{\pi}}^{2^{t-1}}\right]$ corresponds to an attach tuple $A^{(0)}$, and the sum of the entries in that row counts the number of steps of type $\vec{\pi}$ with fixed attach tuple $A^{(0)}$. Therefore, each row of $A\left[\tau_{\vec{\pi}}^{2^{t-1}}\right]$ sums to the same value, so the PerronFrobenius theorem implies that the all-ones vector is the eigenvector associated to $\lambda_{1}\left(A\left[\tau_{\tilde{\pi}}^{2^{t-1}}\right]\right)$. Since $y \otimes \cdots \otimes y$ is perpendicular to the all-ones vector and $A\left[\tau_{\vec{\pi}}^{2^{t-1}}\right]$ is a square real symmetric matrix, $\left|A\left[\tau_{\vec{\pi}}^{2^{t-1}}\right](y \otimes \cdots \otimes y, y \otimes \cdots \otimes y)\right| \leqslant \lambda_{2}\left(A\left[\tau_{\vec{\pi}}^{2^{t-1}}\right)\right.$. Since $|\alpha| \leqslant 1$, the proof is complete.

Proof that $C y C l e_{4 \ell}[\pi] \Rightarrow E i g[\pi]$. Let $\mathcal{H}=\left\{H_{n}\right\}_{n \rightarrow \infty}$ be a sequence of $d$ coregular, $k$-uniform hypergraphs with loops, and let $\tau_{n}$ be the adjacency map of $H_{n}$. For notational convenience, the subscript on $n$ is dropped below. Let $\vec{\pi}$ be any ordering of the entries of $\pi$. Let $m=\left|E\left(C_{\pi, 4 \ell}\right)\right|=2 \ell 2^{t-1}$, and note that $\left|V\left(C_{\pi, 4 \ell}\right)\right|=m k / 2$ since $C_{\pi, 4 \ell}$ is two-regular. The matrix $A=A\left[\tau_{\vec{\pi}}^{2^{t-1}}\right]$ is a square symmetric real-valued matrix, so let $\mu_{1}, \ldots, \mu_{r}$ be the eigenvalues of $A$ arranged so that $\left|\mu_{1}\right| \geqslant \cdots \geqslant\left|\mu_{r}\right|$, where $r=\operatorname{dim}(A)$. The eigenvalues of $A^{2 \ell}$ are $\mu_{1}^{2 \ell}$, $\ldots, \mu_{d}^{2 \ell}$ and the trace of $A^{2 \ell}$ is $\sum_{i} \mu_{i}^{2 \ell}$. Since all $\mu_{i}^{2 \ell} \geqslant 0$, Proposition 6 and Cycle $e_{4 \ell}[\pi]$ imply that

$$
\begin{aligned}
\mu_{1}^{2 \ell} \leqslant \mu_{1}^{2 \ell}+\mu_{2}^{2 \ell} & \leqslant \operatorname{Tr}\left[A^{2 \ell}\right]=\#\left\{\text { possibly degen } C_{\pi, 4 \ell} \text { in } H_{n}\right\} \\
& \leqslant p^{m} n^{m k / 2}+o\left(n^{m k / 2}\right) .
\end{aligned}
$$

Since $p n^{k / 2}=d n^{k / 2-1}=\tau_{\vec{\pi}}(\hat{1}, \ldots, \hat{1}) \leqslant\left\|\tau_{\vec{\pi}}\right\|=\lambda_{1, \pi}(H),(6)$ implies that $\mu_{1} \geqslant$ $p^{2^{t-1}} n^{k 2^{t-2}}$, which implies equality up to $o\left(n^{m k / 2}\right)$ throughout (7). Therefore, $\mu_{2}=$ $o\left(n^{k 2^{t-2}}\right)$, so Corollary 11 shows that $\lambda_{2, \pi}(H)=o\left(n^{k / 2}\right)$, completing the proof.

\section{Acknowledgements}

The authors would like to thank Vojtěch Rödl, Mathias Schacht, and the referees for helpful discussion and feedback. The research of J. Lenz was partly supported by NSA Grant H98230-13-1-0224. The research of D. Mubayi was supported in part by NSF Grants 0969092 and 1300138. 


\section{References}

[1] N. Alon, 'Eigenvalues and expanders', Combinatorica 6(2) (1986), 83-96. Theory of computing (Singer Island, FL, 1984).

[2] N. Alon and V. D. Milman, ' $\lambda_{1}$, isoperimetric inequalities for graphs, and superconcentrators', J. Combin. Theory Ser. B 38(1) (1985), 73-88.

[3] N. Alon and P. Pudlák, 'Constructive lower bounds for off-diagonal Ramsey numbers', Israel J. Math. 122 (2001), 243-251.

[4] N. Alon and V. Rödl, 'Sharp bounds for some multicolor Ramsey numbers', Combinatorica 25(2) (2005), 125-141.

[5] N. Alon and J. H. Spencer, The Probabilistic Method, 3rd edn, Wiley-Interscience Series in Discrete Mathematics and Optimization, (John Wiley \& Sons Inc., Hoboken, NJ, 2008), With an appendix on the life and work of Paul Erdős.

[6] T. Austin and T. Tao, 'Testability and repair of hereditary hypergraph properties', Random Structures Algorithms 36(4) (2010), 373-463.

[7] Y. Bilu and S. Hoory, 'On codes from hypergraphs', European J. Combin. 25(3) (2004), 339-354.

[8] F. Chung, 'Quasi-random hypergraphs revisited', Random Structures Algorithms 40(1) (2012), 39-48.

[9] F. R. K. Chung, 'Quasi-random classes of hypergraphs', Random Structures Algorithms 1(4) (1990), 363-382.

[10] F. R. K. Chung, 'Regularity lemmas for hypergraphs and quasi-randomness', Random Structures Algorithms 2(2) (1991), 241-252.

[11] F. R. K. Chung, 'The Laplacian of a hypergraph', in Expanding Graphs (Princeton, NJ, 1992), DIMACS Series in Discrete Mathematics and Theoretical Computer Science, 10 (American Mathematical Society, Providence, RI, 1993), 21-36.

[12] F. R. K. Chung and R. L. Graham, 'Quasi-random hypergraphs', Random Structures Algorithms 1(1) (1990), 105-124.

[13] F. R. K. Chung and R. L. Graham, 'Quasi-random set systems', J. Amer. Math. Soc. 4(1) (1991), 151-196.

[14] F. R. K. Chung and R. L. Graham, 'Cohomological aspects of hypergraphs', Trans. Amer. Math. Soc. 334(1) (1992), 365-388.

[15] F. R. K. Chung, R. L. Graham and R. M. Wilson, 'Quasi-random graphs', Combinatorica 9(4) (1989), 345-362.

[16] D. Conlon, H. Hàn, Y. Person and M. Schacht, 'Weak quasi-randomness for uniform hypergraphs', Random Structures Algorithms 40(1) (2012), 1-38.

[17] J. Cooper and A. Dutle, 'Spectra of uniform hypergraphs', Linear Algebra Appl. 436(9) (2012), 3268-3292.

[18] P. Erdős and A. Hajnal, 'On Ramsey like theorems. Problems and results', in Combinatorics (Proc. Conf. Combinatorial Math., Math. Inst., Oxford, 1972) (Inst. Math. Appl., Southend, 1972), 123-140.

[19] K. Feng and W.-C. W. Li, 'Spectra of hypergraphs and applications', J. Number Theory 60(1) (1996), 1-22.

[20] P. Frankl and V. Rödl, 'The uniformity lemma for hypergraphs', Graphs Combin. 8(4) (1992), 309-312.

[21] J. Friedman, 'Some graphs with small second eigenvalue', Combinatorica 15(1) (1995), $31-42$. 
[22] J. Friedman and A. Wigderson, 'On the second eigenvalue of hypergraphs', Combinatorica 15(1) (1995), 43-65.

[23] W. T. Gowers, 'Quasirandomness, counting and regularity for 3-uniform hypergraphs', Combin. Probab. Comput. 15(1-2) (2006), 143-184.

[24] W. T. Gowers, 'Hypergraph regularity and the multidimensional Szemerédi theorem', Ann. of Math. (2) 166(3) (2007), 897-946.

[25] W. T. Gowers, 'Quasirandom groups', Combin. Probab. Comput. 17(3) (2008), 363-387.

[26] S. Hoory, N. Linial and A. Wigderson, 'Expander graphs and their applications', Bull. Amer. Math. Soc. (N.S.) 43(4) (2006), 439-561. (electronic).

[27] P. Keevash, 'A hypergraph regularity method for generalized Turán problems', Random Structures Algorithms 34(1) (2009), 123-164.

[28] Y. Kohayakawa, B. Nagle, V. Rödl and M. Schacht, 'Weak hypergraph regularity and linear hypergraphs', J. Combin. Theory Ser. B 100(2) (2010), 151-160.

[29] Y. Kohayakawa, V. Rödl and J. Skokan, 'Hypergraphs, quasi-randomness, and conditions for regularity', J. Combin. Theory Ser. A 97(2) (2002), 307-352.

[30] M. Krivelevich and B. Sudakov, 'Pseudo-random graphs', in More Sets, Graphs and Numbers, Bolyai Society Mathematical Studies, 15 (Springer, Berlin, 2006), 199-262.

[31] J. Lenz and D. Mubayi, 'Eigenvalues and linear quasirandom hypergraphs', online at http://arxiv.org/abs/1208.4863.

[32] J. Lenz and D. Mubayi, 'Eigenvalues of non-regular linear quasirandom hypergraphs', online at http://www.math.uic.edu/ lenz/nonregular-art.pdf.

[33] J. Lenz and D. Mubayi, 'Multicolor Ramsey numbers for complete bipartite versus complete graphs', J. Graph Theory 77(1) (2014), 19-38.

[34] J. Lenz and D. Mubayi, 'The poset of hypergraph quasirandomness', Random Structures and Algorithms http://arxiv.org/abs/1208.5978 (accepted).

[35] L. Lu and X. Peng, 'High-ordered random walks and generalized Laplacians on hypergraphs', in Algorithms and Models for the Web Graph, Lecture Notes in Computer Science, 6732 (Springer, Heidelberg, 2011), 14-25.

[36] L. Lu and X. Peng, 'Loose Laplacian spectra of random hypergraphs', Random Structures Algorithms 41(4) (2012), 521-545.

[37] M. G. Martínez, 'The finite upper half space and related hypergraphs', J. Number Theory 84(2) (2000), 342-360.

[38] M. G. Martínez, H. M. Stark and A. A. Terras, 'Some Ramanujan hypergraphs associated to $\operatorname{GL}\left(n, \mathbb{F}_{q}\right)$ ', Proc. Amer. Math. Soc. 129(6) (2001), 1623-1629. (electronic).

[39] A. Steger, Die Kleitman-Rothschild Methode. PhD thesis, Forschungsinstitut für Diskrete Mathematik, Rheinische Friedrichs-Wilhelms-Universität Bonn, March 1990.

[40] C. K. Storm, 'The zeta function of a hypergraph', Electron. J. Combin. 13(1) (2006), 26 Research Paper 84 (electronic).

[41] T. Szabó, 'On the spectrum of projective norm-graphs', Inform. Process. Lett. 86(2) (2003), 71-74.

[42] R. M. Tanner, 'Explicit concentrators from generalized N-gons', SIAM J. Algebra Discrete Meth. 5(3) (1984), 287-293.

[43] A. Thomason, 'Pseudorandom graphs', in Random graphs '85 (Poznań, 1985), NorthHolland Math. Stud., 144 (North-Holland, Amsterdam, 1987), 307-331.

[44] A. Thomason, 'Random graphs, strongly regular graphs and pseudorandom graphs', in Surveys in Combinatorics 1987 (New Cross, 1987), London Mathematical Society Lecture Note Series, 123 (Cambridge University Press, Cambridge, 1987), 173-195. 